\title{
Long-term cyclic persistence in an experimental predator-prey system
}

https://doi.org/10.1038/s41586-019-1857-0

Received: 2 July 2019

Accepted: 18 November 2019

Published online: 18 December 2019

\section{Bernd Blasius $^{1,2 *}$, Lars Rudolf ${ }^{3}$, Guntram Weithoff ${ }^{4,5}$, Ursula Gaedke ${ }^{4,5}$ \& Gregor F. Fussmann ${ }^{6}$}

Predator-prey cycles rank among the most fundamental concepts in ecology, are predicted by the simplest ecological models and enable, theoretically, the indefinite persistence of predator and prey $^{1-4}$. However, it remains an open question for how long cyclic dynamics can be self-sustained in real communities. Field observations have been restricted to a few cycle periods ${ }^{5-8}$ and experimental studies indicate that oscillations may be short-lived without external stabilizing factors ${ }^{9-19}$. Here we performed microcosm experiments with a planktonic predator-prey system and repeatedly observed oscillatory time series of unprecedented length that persisted for up to around 50 cycles or approximately 300 predator generations. The dominant type of dynamics was characterized by regular, coherent oscillations with a nearly constant predator-prey phase difference. Despite constant experimental conditions, we also observed shorter episodes of irregular, non-coherent oscillations without any significant phase relationship. However, the predator-prey system showed a strong tendency to return to the dominant dynamical regime with a defined phase relationship. A mathematical model suggests that stochasticity is probably responsible for the reversible shift from coherent to non-coherent oscillations, a notion that was supported by experiments with external forcing by pulsed nutrient supply. Our findings empirically demonstrate the potential for infinite persistence of predator and prey populations in a cyclic dynamic regime that shows resilience in the presence of stochastic events.
Cyclic dynamics are one of the most notable phenomena in population biology and are known to occur in a large range of communities both in the wild ${ }^{5-8,13,14}$ and the laboratory ${ }^{9-12,15-18}$. A number of mechanisms that cause populations to oscillate have previously been identified ${ }^{3,4,20}$; the most highly investigated, however, are the cyclic dynamics that arise from trophic interactions between populations of predator and prey organisms. The nearly century-old fascination with this type of dynamics is rooted in the predictions of simple mathematical models, which suggest that the predator and prey may coexist on recurring cyclic trajectories over indefinitely long periods of time $\mathrm{e}^{1,2}$. Empirical data that support the sustained nature of predator-prey cycles in the field are difficult to come by because tractable populations typically have cycle periods of three to ten years ${ }^{4,5,13}$. An alternative data source are laboratory studies with fast-reproducing organisms, with which long-running experiments can be realized under strictly controlled conditions ${ }^{9,10,12,16,21}$. In a seminal experimental study, population numbers of weevils and their larval parasites were recorded for a time span of about 20 cycles but oscillations could not be sustained for the whole duration of the experiment ${ }^{10}$. This observation is paradigmatic for many studies, which showed that cycles in closed laboratory predator-prey systems tend to be short-lived, with populations either becoming extinct or reaching a stationary state.

These findings suggest that sustained, intrinsically generated predator-prey cycles might be a construct of ecological theory and that predator-prey systems require external drivers such as spatial structure, immigration or environmental perturbations to exhibit persistent cycles $^{9,11,12,17,22,23}$. To explore the potential for long-term persistence of predator-prey cycles, we cultured freshwater organisms-planktonic rotifers together with their prey, unicellular green algae-in several independent chemostat trials under constant environmental conditions (Methods, experiments $\mathrm{C} 1-\mathrm{C} 7$ ). One of our experimental systems (Fig. 1a and Extended Data Fig. 1; C1 in Extended Data Table 1) persisted without any external stimuli in a homogeneous environment for over a year and displayed more than 50 cycles, which is equivalent to about 300 predator generations and at least as many prey generations. This result of the long-term persistence of the predator and prey in a cyclic dynamic regime was confirmed in four additional, replicated chemostat experiments with the same species (C2-C5 in Extended Data Table 1, Extended Data Fig. 2), and in two additional experiments (C6 and C7) with a different algal prey species (Extended Data Fig. 3). We applied power analysis and phase analysis (in particular, bivariate wavelet analysis ${ }^{21,24-26}$ ) (Methods) to quantify the cyclic succession and the distribution of phase differences in our measured time series. Wavelet analysis revealed statistically significant associations between the dynamics of predator and prey densities in our experiments and power analysis determined a mean period length of 6.7 days (C1-C5) (Fig. 1d and Extended Data Fig. 1d, f).

${ }^{1}$ Institute for Chemistry and Biology of the Marine Environment ICBM, University of Oldenburg, Oldenburg, Germany. ${ }^{2} \mathrm{Helmholtz}$ Institute for Functional Marine Biodiversity, University of Oldenburg (HIFMB), Oldenburg, Germany. ${ }^{3}$ Institute of Physics and Astronomy, University of Potsdam, Potsdam, Germany. ${ }^{4}$ Department of Ecology \& Ecosystem Modelling, University of Potsdam, Potsdam, Germany. ${ }^{5}$ Berlin-Brandenburg Institute for Advanced Biodiversity Research, Berlin, Germany. ${ }^{6}$ Department of Biology, McGill University, Montréal, Québec, Canada. *e-mail: blasius@icbm.de 

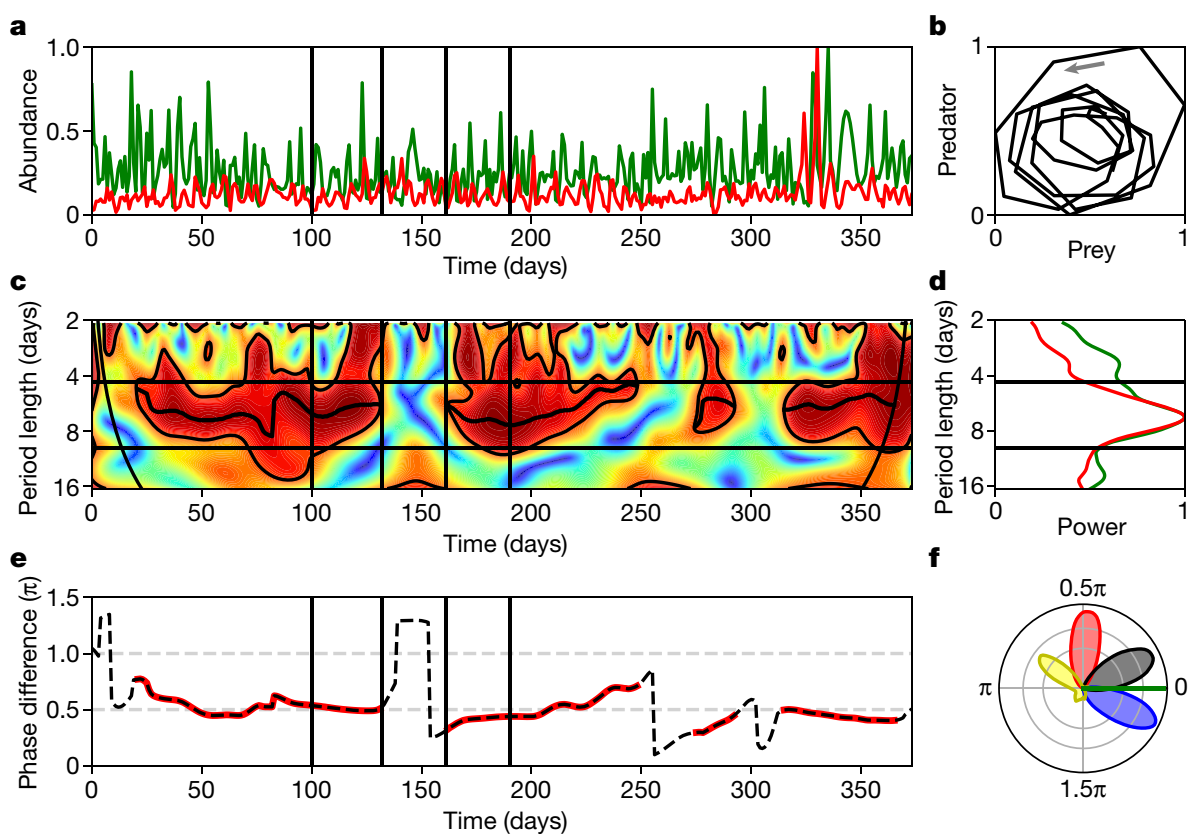

Fig. 1 Phase analysis of a year-long, oscillatory predator-prey time series (experiment C1).a, Time series of the predator, B. calyciflorus (red) and its prey, Monoraphidium minutum (green); abundance normalized to a range of 0 to 1.b, Phase portrait of the bandpass-filtered predator-prey time series between days 90 and 135; the arrow indicates the direction of oscillation. c, Wavelet coherence (WCO) between predator and prey. Values range from 0 (blue) to 1 (red). The cone of influence is indicated by black thin curved lines. The black contour lines enclose significant areas (95\% significance level, WCO $>0.83$ ). The thick black line segments show the instantaneous oscillation period $\widetilde{s}(t)$ of highest WCO within these areas (coherent oscillation regimes). d, Global wavelet power spectrum $P(s)$ for the predator (red) and the prey (green).e, Phase difference $\theta(t)$ between predator and prey; well-defined relations between both signals are marked in red.f, Circular distribution of the mutual phase difference to prey (indicated in green) for the predator (red), the predator egg ratio (blue), the number of eggs (black) and the dead animals (yellow).c, d, The two horizontal lines indicate the prefixed period band of $\left[s_{1}, s_{2}\right] . \mathbf{a}, \mathbf{c}, \mathbf{e}$, The solid vertical lines indicate selected time spans of regular and irregular oscillations, which are enlarged in Fig. 2.

\section{Transitions between different oscillation regimes}

Although the experiments were not perturbed by external influences, we identified sharp transitions between two different dynamic regimes. For most of the time, we observed oscillations with a significantly coherent, nearly constant phase relationship (coherent oscillation regimes) (Methods), in which the predator densities followed the prey densities with a phase lag of about $\theta=0.5 \pi$ (equivalent to $90^{\circ}$ ), consistent with classical predator-prey theory. These periods with coherent oscillations were intersected by non-coherent oscillation regimes in which the well-defined phase relationship between the measured signals was lost, but re-initiated shortly after (Fig. 1c, e). For example, the time interval between days 100 and 131 in C1 (Fig. 2a, b) and between days 37 and 66 in C7 (Fig. 2c, d) showed sustained cycles with regular period lengths and a constant phase lag between prey and predator densities, which is also reflected in the anticlockwise motion in the prey-predator phase plane (Fig. 1b). This regime of coherent oscillations gave way to a noncoherent, more irregular time series (days 132-161 in C1 and days 67-97 in C7), in which the phase relationship between prey and predator was lost, although both populations continued to oscillate (Fig. 2). Finally, and without external intervention, the system displayed resilience and the characteristic predator-prey phase difference was re-established.

Combining the analyses of all five experiments with the same predator and prey species (C1-C5), we found that coherent oscillations were the dominant type of dynamics and comprised $66 \%$ of the total experimental time with a typical regime duration of 58 days (Extended Data Fig. 4 and Methods). In the remaining $34 \%$ of the experiments, predator and prey continued to oscillate, but without a well-defined phase relationship (typical duration of non-coherent regimes, 23 days) (Methods). Phase analysis of surrogate data revealed that spurious coherent oscillation regimes can arise also by chance, but with a significantly smaller duration (typical duration, 15 days) (Extended Data Fig. 4).
We performed additional analyses and experiments that were designed to provide mechanistic insights into the origin of the observed coherent oscillations and the intermittent breaks in the phase signature. First, we extended the specificity of the phase analysis by including predator life-history stages. Second, we formulated and analysed a mathematical model that accounts for the predator's stage structure. And third, we performed three additional chemostat experiments: two experiments with periodic changes in the nutrient concentration in the supply medium, to explore how the phase relations were influenced by external forcing (C8 and C9); and one experiment at dilution rates that, according to bifurcation theor $y^{2,16}$ and our mathematical model, should cause the predator-prey system to cross over from oscillatory to equilibrium dynamics (C10).

\section{Phase analysis of life-history stages}

The predator in our system, the rotifer Brachionus calyciflorus ${ }^{27}$, is a small freshwater metazoan that reproduces asexually in the chemostats (only females occur) and undergoes a stage-structured life cycle. Reproducing females carry $1-5$ eggs, from which juveniles hatch and grow to adulthood without any larval stages; adults die shortly after the last egg has hatched. In addition to analysing algal and rotifer densities, we applied phase analysis to all discernible predator life-history stages: the number of eggs, the egg ratio (that is, the average number of eggs per female rotifer) and the number of dead animals. We found that these life-history parameters also exhibited persistent cycles that were coherently locked in phase with prey abundance (Fig. 3a). These phase differences were notably constant over all different experimental replicates (Extended Data Figs. 1-3). Averaging across all experiments (C1-C5), we obtained the following phase signature: the predator density followed the prey density with a phase difference of $93^{\circ} \pm 21^{\circ}$, the 

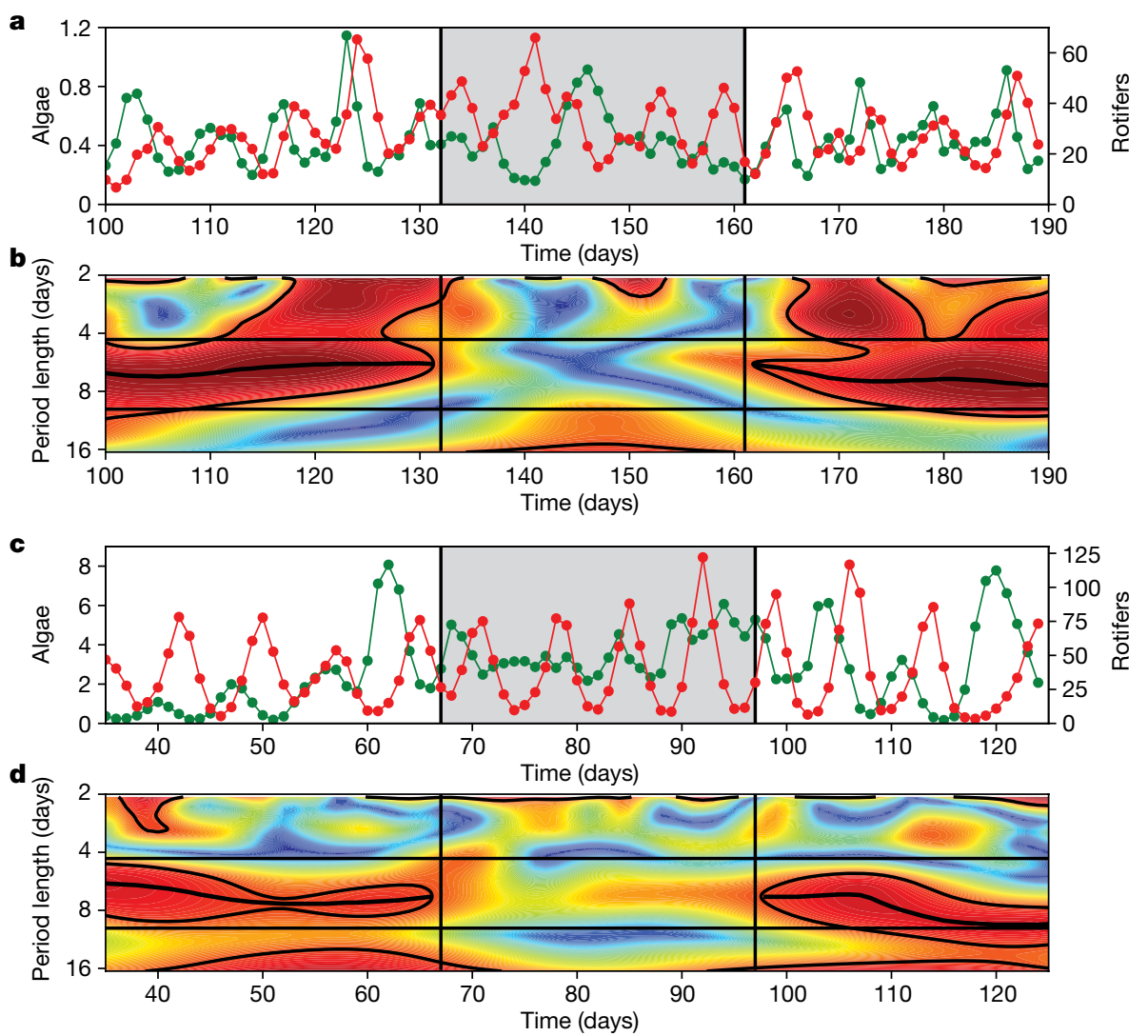

Fig. 2 | Resilience of phase relationship in experiments with two different prey species. a, Enlarged view of the densities of the predator (animals per millilitre, red) and prey (M. minutum, $10^{6}$ cells per millilitre, green) in experiment $\mathrm{C} 1$ for a time window of 90 days. b, Enlarged view of their WCO during the same time interval (colours and lines as in Fig. 1c).a, b, Solid vertical lines border a time interval without significant wavelet coherence between predator and prey, separating three dynamic regimes: days 100-131, distinct predator-prey cycles with a constant phase shift of $\theta=\pi / 2$; days 132-161, regime of non-coherent oscillations (grey background); days 162-190, spontaneous re-initiation of the regular phase-locked oscillation. $\mathbf{c}, \mathbf{d}$, As in a, b, but showing a time window of 90 days for experiment $\mathrm{C7}$ with a different algal prey, Chlorella vulgaris. Non-coherent oscillations arise between days 67 and 97. abundance of eggs was delayed to the abundance of prey with a phase difference of $27^{\circ} \pm 19^{\circ}$, whereas the egg ratio preceded the prey signal $\left(-27^{\circ} \pm 16^{\circ}\right)$; dead animals had a phase difference of $-160^{\circ} \pm 68^{\circ}$. These mutual phase differences can be compactly visualized in a single circular histogram $^{21}$ (Figs. If and 3b). This 'phase signature' enables the straightforward interpretation of the temporal succession of the community during the cycles and thus provides a fingerprint for the cyclic community structure.

\section{Mathematical model}

We compared our experimental results with numerical simulations of a stage-structured predator-prey model that included the operative parameters of the chemostat (inflow nutrient concentration and dilution rate) and essential organismal variables (algae as well as rotifer eggs, juveniles, adults and dead animals) (Extended Data Fig. 5 and Methods). Numerical simulations revealed sustained oscillations with a period length of 6.8 days (experimental mean, 6.7 days). When we introduced stochasticity into the model, we observed similar breaks in the oscillation regimes as in experiments $\mathrm{C} 1-\mathrm{C} 7$, with random transitions between coherent and non-coherent oscillating regimes that were triggered by stochasticity alone. We were still able to recover a phase signature that aligned well with the experimental observations (predator, $90^{\circ} \pm 11^{\circ}$; eggs, $11^{\circ} \pm 13^{\circ}$; egg ratio, $-21^{\circ} \pm 2^{\circ}$; dead rotifers, $-150^{\circ} \pm 60^{\circ}$ ) (Fig. $3 \mathrm{c}$, d and Extended Data Fig. 5).

Notably, both in the data and the model, the predator egg ratio preceded the phase of prey availability, which is not immediately obvious as egg production is directly connected to food availability. This phase lag between prey density and egg ratio disappeared in model simulations when the juvenile maturation rate was set to zero (Extended Data Fig. 6), revealing the effect of juveniles on the phase difference signature. Juvenile predators (which, as immature individuals, carry no eggs by definition) have a crucial influence as they lead to an early breakdown of the egg-ratio maximum, caused by the multitude of non-egg-bearing juveniles during the prey maximum, finally giving rise to a negative phase difference between egg ratio and prey (Fig. 3a, c and Extended Data Fig. 6a). Thus, as physiological information may be encoded in the phase difference distributions, extending phase analysis to life-history stages enabled deeper insights into the functioning of our system.

\section{Additional experiments}

In experiments C8 and C9, we externally forced the dynamics through pulsed supply of nutrients (Methods). We chose a forcing period (8.0 days) that was clearly distinguishable from the period of the unforced experiments (6.7 days) but sufficiently close to enable, in theory, predator and prey (that is, living populations with demographic constraints) to lock to the external signal. Indeed, we observed predator-prey oscillations that were locked to the external signal, both in chemostat experiments and model results (Fig. $3 \mathrm{e}, \mathrm{g}$ and Extended Data Fig. 7). As expected, oscillations were persistently coherent, without the non-coherent breaks in the time series from the unforced experiments $(\mathrm{C} 1-\mathrm{C} 7)$, although intermittent period doubling occurred in experiment C8 (Fig. 3e and Extended Data 

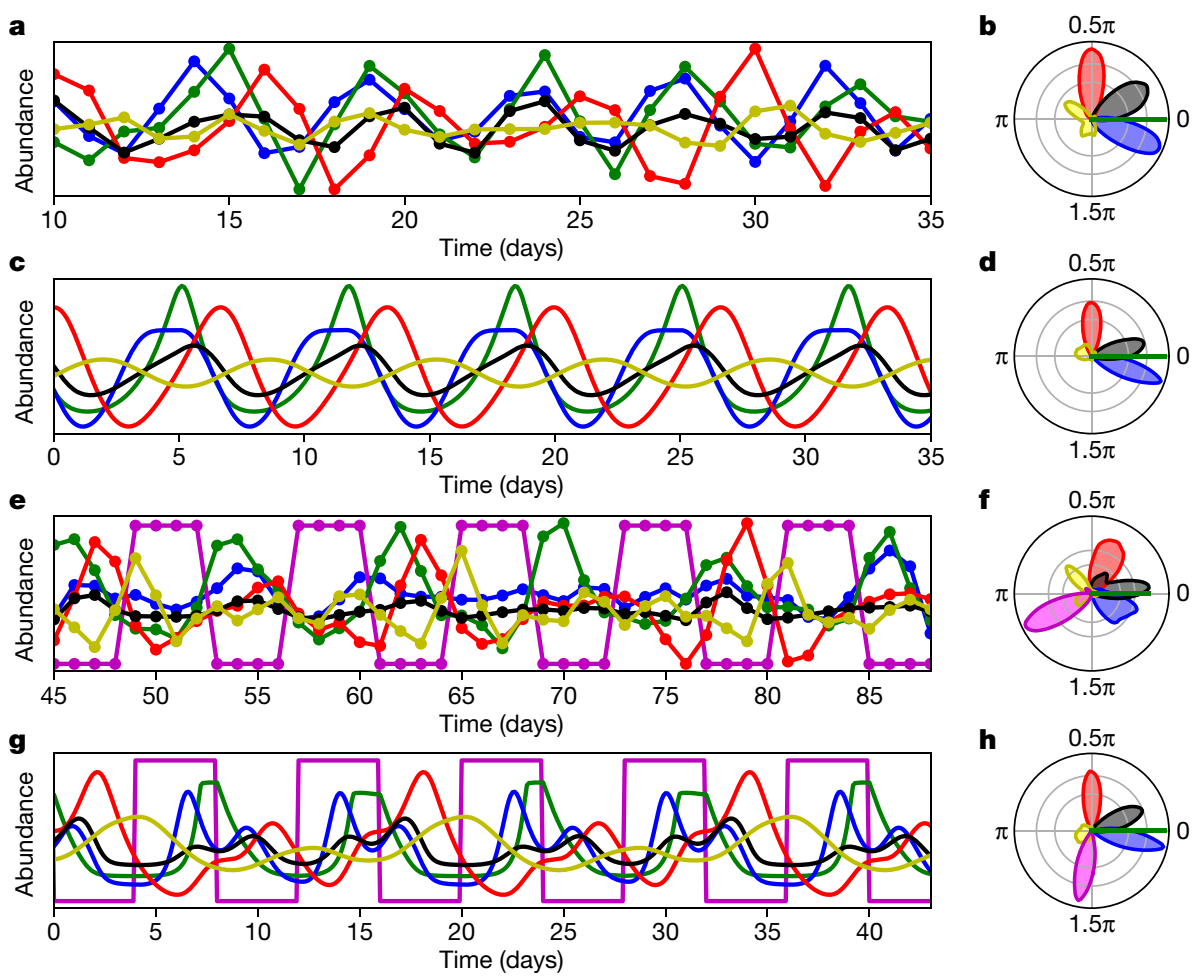

Fig. 3 | Dynamics and phase relationships in unforced versus externally forced systems. a, Time series of algae (green), rotifers (red), eggs (black), egg ratio (blue) and dead animals (yellow) in an unforced (that is, 'free-running' under constant conditions) experiment covering five oscillations (days 10-35 in C2). Data were filtered with a second order Butterworth bandpass filter and are shown in relative units. $\mathbf{b}$, Phase signature aggregated over all experiments with the algal prey M. minutum under unforced conditions (C1-C5, including a total duration of 965 days), showing the circular distribution of the mutual phase differences to prey (colour code as in a).c, Simulated time series from the predator-prey model under constant conditions with all stochasticity removed (mean centred, relative units). d, Phase signature obtained from stochastic simulations of the predator-prey model reproduces the experimentally observed phase relationships and variances in b.e, As in a, but for an experiment with external forcing (days $45-88$ of C8). The concentration of the external medium is shown in magenta.f, Phase signature aggregated over all experiments with external forcing (C8-C9, including a total duration of 323 days). $\mathbf{g}, \mathbf{h}$, As in c, $\mathbf{d}$, but for simulations in an externally forced system.
Fig. 7c, d). Notably, externally forced and unforced experiments produced essentially the same phase signature (Fig. 3b, f), which was repeated also in model simulations (Fig. 3d, h). Thus, even in a dynamic regime that was strongly influenced by and locked to an external factor, phase analysis of different life-history stages was able to reveal the underlying predator-prey structure and to disentangle between intrinsic and external origins of cycles. This demonstrates that phase signature analysis can be a powerful diagnostic tool for the analysis of field data, for which it is often not known whether oscillations are the result of external factors or originate from species interactions.

Finally, we confirmed that the coherent oscillations observed in our experimental system indeed represent stable limit cycles, which arise from the destabilization of a stable equilibrium point. Oscillations in experiment $\mathrm{C} 10$ persisted only in a certain parameter range; if the dilution rate was increased, the oscillations died out (Extended Data Fig. 8). This result agrees with model predictions and previous experiments showing that increased dilution rates drive the system over a Hopf bifurcation ${ }^{16}$ (Extended Data Fig. 9).

\section{Conclusions}

Persistent and coherent predator-prey oscillations are a potential dynamic regime enabling the prolonged coexistence of predator and prey populations, as predicted by fundamental theory. In live systems, stochasticity and other external perturbations can obscure or prevent predator-prey oscillations, and our simulations suggest that stochasticity is probably responsible for the reversible shifts from coherent to more-erratic oscillations that we observed in our highly controlled experiments. Four separate lines of evidence support a causal relationship between sustained cycles and predator-prey interactions. (1) Cyclic patterns dominated throughout the entire experiment and the system displayed resilience-that is, it returned to a well-defined phase relationship after intermittent non-coherent periods. (2) Planned experimental interventions enabled us to suppress cycles (C10) and to quench intermittent non-coherent oscillations (C8 and C9). (3) Data on the stage structure of the predator population enabled us to explicitly link characteristics of the predator-prey cycles to recurring changes in the demographic structure of the predator population. Therefore, our case for sustained predator-prey cycles is much stronger than it is possible for unstructured microbial populations, in which population densities are the only variables. (4) Finally, the experimental predator-prey cycles manifested as a regular phase-locked succession, a pattern that we were able to capture as a community phase signature. Our approach firmly establishes this phase signature-which, in a similar way, can be constructed for any oscillating system-as a robust measure for identifying species interactions, cyclic or seasonal species succession, and the regulatory dependencies that underlie community dynamics.

\section{Online content}

Any methods, additional references, Nature Research reporting summaries, source data, extended data, supplementary information, acknowledgements, peer review information; details of author contributions and competing interests; and statements of data and code availability are available at https://doi.org/10.1038/s41586-019-1857-0. 


\section{Article}

1. Volterra, V. Fluctuations in the abundance of a species considered mathematically. Nature 118, 558-560 (1926).

2. May, R. M. Limit cycles in predator-prey communities. Science 177, 900-902 (1972)

3. Kendall, B. E. et al. Why do populations cycle? A synthesis of statistical and mechanistic modeling approaches. Ecology 80, 1789-1805 (1999).

4. Berryman, A. A. (ed.) Population Cycles: The Case for Trophic Interactions (Oxford Univ. Press, 2002).

5. Elton, C. \& Nicholson, M. The ten-year cycle in numbers of the lynx in Canada. J. Anim. Ecol. 11, 215-244 (1942).

6. Blasius, B., Huppert, A. \& Stone, L. Complex dynamics and phase synchronization in spatially extended ecological systems. Nature 399, 354-359 (1999).

7. Gilg, O., Hanski, I. \& Sittler, B. Cyclic dynamics in a simple vertebrate predator-prey community. Science 302, 866-868 (2003).

8. Ryabov, A. B., de Roos, A. M., Meyer, B., Kawaguchi, S. \& Blasius, B. Competition-induced starvation drives large-scale population cycles in Antarctic krill. Nat. Ecol. Evol. 1, 0177 (2017).

9. Gause, G. F., Smaragdova, N. P. \& Witt, A. A. Further studies of interaction between predators and prey. J. Anim. Ecol. 5, 1-18 (1936).

10. Utida, S. Cyclic fluctuations of population density intrinsic to the host-parasite system. Ecology 38, 442-449 (1957).

11. Huffaker, C. B. Experimental studies on predation: dispersion factors and predator-prey oscillations. Hilgardia 27, 343-383 (1958).

12. Luckinbill, L. S. Coexistence in laboratory populations of Paramecium aurelia and its predator Didinium nasutum. Ecology 54, 1320-1327 (1973).

13. Krebs, C. J. et al. Impact of food and predation on the snowshoe hare cycle. Science $\mathbf{2 6 9}$ $1112-1115$ (1995).

14. Hudson, P. J., Dobson, A. P. \& Newborn, D. Prevention of population cycles by parasite removal. Science 282, 2256-2258 (1998).

15. McCauley, E., Nisbet, R. M., Murdoch, W. W., de Roos, A. M. \& Gurney, W. S. C. Largeamplitude cycles of Daphnia and its algal prey in enriched environments. Nature 402, 653-656 (1999).

16. Fussmann, G. F., Ellner, S. P., Shertzer, K. W. \& Hairston, N. G. Jr. Crossing the Hopf bifurcation in a live predator-prey system. Science 290, 1358-1360 (2000).
17. Ellner, S. P. et al. Habitat structure and population persistence in an experimental community. Nature 412, 538-543 (2001).

18. Becks, L., Hilker, F. M., Malchow, H., Jürgens, K. \& Arndt, H. Experimental demonstration of chaos in a microbial food web. Nature 435, 1226-1229 (2005).

19. Massie, T. M., Blasius, B., Weithoff, G., Gaedke, U. \& Fussmann, G. F. Cycles, phase synchronization, and entrainment in single-species phytoplankton populations. Proc. Natl Acad. Sci. USA 107, 4236-4241 (2010).

20. Radchuk, V., Ims, R. A. \& Andreassen, H. P. From individuals to population cycles: the role of extrinsic and intrinsic factors in rodent populations. Ecology 97, 720-732 (2016).

21. Benincà, E., Jöhnk, K. D., Heerkloss, R. \& Huisman, J. Coupled predator-prey oscillations in a chaotic food web. Ecol. Lett. 12, 1367-1378 (2009)

22. Holyoak, M. \& Lawler, S. P. Persistence of an extinction-prone predator-prey interaction through metapopulation dynamics. Ecology 77, 1867-1879 (1996).

23. Stenseth, N. C. et al. The effect of climatic forcing on population synchrony and genetic structuring of the Canadian lynx. Proc. Natl Acad. Sci. USA 101, 6056-6061 (2004).

24. Torrence, C. \& Compo, G. P. A practical guide to wavelet analysis. Bull. Am. Meteorol. Soc. 79, 61-78 (1998).

25. Cazelles, B. et al. Wavelet analysis of ecological time series. Oecologia 156, 287-304 (2008).

26. Keitt, T. H. Coherent ecological dynamics induced by large-scale disturbance. Nature 454, 331-334 (2008).

27. Paraskevopoulou, S., Tiedemann, R. \& Weithoff, G. Differential response to heat stress among evolutionary lineages of an aquatic invertebrate species complex. Biol. Lett. 14, 20180498 (2018).

28. Guillard, R. R. L. \& Lorenzen, C. J. Yellow-green algae with chlorophyllide c. J. Phycol. 8, 10-14 (1972).

Publisher's note Springer Nature remains neutral with regard to jurisdictional claims in published maps and institutional affiliations.

(c) The Author(s), under exclusive licence to Springer Nature Limited 2019 


\section{Methods}

\section{Data reporting}

No statistical methods were used to predetermine sample size. The experiments were not randomized and the investigators were not blinded to allocation during experiments and outcome assessment.

\section{Experiments}

We ran chemostat experiments with parthenogenetic rotifers ( $B$. calyciflorus sensu strictu ${ }^{27}$, a small metazoan freshwater zooplankton species) as predators and unicellular algae (M. minutum or C.vulgaris) as prey under constant temperature $\left(23^{\circ} \mathrm{C}\right)$ and permanent illumination. Experiments were conducted (mostly consecutively) in the same well-maintained climate chamber. We carefully searched for possible sources of external perturbations, but all measured parameters (for example, temperature and irradiance) remained constant throughout the experimental runs. For inoculation, we used stock cultures originally raised from a single individual and added $B$. calyciflorus 10 days after the algae had reached a biomass that enabled rotifer growth. The chemostats (experimental volume, $0.8 \mathrm{l}$ ) had a constant inflow of sterile medium with a rate of 0.55 per day. We used a modified WC medium ${ }^{28}$ with a reduced nitrogen concentration of $80 \mu \mathrm{moll}^{-1}$ nitrate as the limiting resource. In the experiments with external forcing, we periodically changed the concentration in the external medium to between 0 and $160 \mu \mathrm{mol} \mathrm{I}^{-1}$ nitrate with a period length of 8 days. We took daily subsamples of $8 \mathrm{ml}$ to determine the abundance of both predator and prey. The algal abundance was analysed with an electronic particle counter (CASY, Schärfe). The rotifers were counted using an inverted microscope at 100-fold magnification. For the rotifers, we recorded the total number of rotifers and of asexually produced subitaneous eggs and the dead animals. No males or sexually reproducing females were observed throughout the experiment. The egg ratio (that is, the total number of eggs divided by the total number of animals in the population) is a measure of the reproductive status of the population.

\section{Bivariate wavelet analysis}

Because the measured signals showed large variability, we applied phase analysis ${ }^{6}$, which relies on the fact that the regulatory dependence between state variables is often encoded in their phase relationship, whereas the amplitudes may be highly erratic and uncorrelated ${ }^{6}$. The wavelet method ${ }^{24}$ is a particularly powerful tool for extracting optimally resolved phase information from ecological time series ${ }^{25,26}$ and enables us to quantify transient associations between two nonstationary signals ${ }^{24,29-32}$.

\section{Continuous wavelet transformation}

The continuous wavelet transformation ${ }^{24}$ (CWT) of a signal $x(t)$ is calculated as the convolution of the signal with a localized complexvalued wavelet function $\psi(\tau)$ centred at time $t$ and dilated by the scale parameter $s$

$$
W_{x}(s, t)=\frac{1}{\sqrt{s}} \int \mathrm{d} t^{\prime} x\left(t^{\prime}\right) \psi^{*}\left(\frac{t^{\prime}-t}{s}\right)
$$

Here, the asterisk denotes the complex conjugate. After decomposing the complex CWT into amplitude and phase $W_{x}(s, t)=\left|W_{x}(s, t)\right| \mathrm{e}^{i \phi_{x}(s, t)}$ intuitively it can be understood as the extent $\left|W_{x}(s, t)\right|$ to which the signal $x(t)$ at local time $t$ resembles an oscillation with period length $s$ and phase $\phi_{x}(s, t)$.

Throughout our analysis, we used the Morlet wavelet $\psi(\tau)=$

$\pi^{-1 / 4} \mathrm{e}^{i \omega_{0} \tau} \mathrm{e}^{-\tau^{2} / 2}$ with $\omega_{0}=6$ so that the scale parameter $s$ can simply be interpreted as the period length of the oscillation ${ }^{24}$. We always took $s$ from 100 uniform steps per octave on a logarithmic scale. Edge effects were minimized by applying zero padding to the time series to the next highest integer power of two. We always indicate the cone of influence, which marks the zone in the CWT that is affected by edge effects ${ }^{24}$.

\section{Wavelet power spectrum}

Although the wavelet transform is complex, the real-valued local wavelet power spectrum (WPS) of a signal $x(t)$ at time $t$ and scale $s$ can be defined as

$$
W_{x x}(s, t)=\left\langle W_{x}(s, t) W_{x}^{*}(s, t)\right\rangle
$$

in which '〈... 〉' denotes a smoothing operator in both scale and time (see 'Parametrization and smoothing'). The global WPS $P_{x}(s)$ is defined as the time average of the local WPS and measures the averaged variance of the signal $x(t)$ at scale $s$. We used the maximum of $P_{x}(s)$ to estimate the mean oscillation period of the signal.

\section{Wavelet cross-spectrum}

To quantify the statistical relationship between two non-stationary signals $x(t)$ and $y(t)$, the wavelet cross-spectrum (WCS) is defined as the smoothed product of the corresponding wavelet transforms ${ }^{24,29-31}$

$$
W_{x, y}(s, t)=\left\langle W_{x}(s, t) W_{y}^{*}(s, t)\right\rangle
$$

The WCS is complex-valued. After decomposition into amplitude and phase $W_{x, y}(s, t)=A_{x, y}(s, t) \mathrm{e}^{i \Delta \phi_{x, y}(s, t)}$, the phase describes the de-lay $\Delta \phi_{x, y}(s, t)=\phi_{x}(s, t)-\phi_{y}(s, t)$ between the two signals at time $t$ and scale $s$, whereas the amplitude $A_{x, y}(s, t)$ expresses the covarying power of the two processes.

\section{Wavelet coherence}

The WCS is not specific, because it exhibits high values when either a true covariance between the two signals exists or when one of the spectra exhibits a high value. To solve this problem, a normalized measure is given by the $\mathrm{WCO}^{29-31}$, which is defined as the amplitude of the WCS divided by the square roots of the two local WPS

$$
\mathrm{WCO}_{x, y}(s, t)=\frac{\left|W_{x, y}(s, t)\right|}{W_{x, x}(s, t)^{1 / 2} W_{y, y}(s, t)^{1 / 2}}
$$

The WCO estimates the relationship between the two signals at time $t$ and scale $s$, normalized into the range $0 \leq \mathrm{WCO} \leq 1$. As the WCO is realvalued, it cannot be used to extract the delay between two signals, in contrast to the WCS.

\section{Dominant phase difference}

The phase difference $\Delta \phi_{x, y}(s, t)$ between two signals $x(t)$ and $y(t)$, as obtained from the WCS, depends on the scale parameter $s$. Assume now that the two signals oscillate with a common, but possibly timevarying, period length. To obtain their dominant phase difference, we define for every time instance $t$ the dominant scale $\tilde{s}(t)$ as the scale parameter that has the maximal WCO at time $t$ over all scales in a prefixed band $\left[s_{1}, s_{2}\right]$ :

$$
\tilde{s}(t)=\operatorname{argmax}_{s_{1}<s<s_{2}} \mathrm{WCO}(s, t)
$$

These values $\tilde{s}(t)$ constitute a line of the strongest co-oscillating component of both signals in the timescale plane ${ }^{32}$. Inserting this line in the WCS yields the dominant phase difference $\theta_{x, y}(t)$ between the two signals at every time instance

$$
\theta_{x, y}(t)=\phi_{x}(\tilde{s}(t), t)-\phi_{y}(\tilde{s}(t), t)
$$

\section{Significance testing}

To assess the statistical significance of the WCO, we generated $n=1,000$ independent realizations of surrogate data by time-scrambling the 
empirical signals (that is, sampling 1,000 random values with replacement from the measured predator and prey time series). The resulting surrogate data retain the frequency histogram of the original data but lose all temporal correlations. Using Monte Carlo simulations, we found that for our chosen parameterization (see 'Parametrization and smoothing') two signals are significantly correlated at time $t$ and scale $s$ on a $95 \%$ critical level if $\mathrm{WCO}(s, t)=0.83$.

\section{Coherent oscillation regime}

We defined a maximal contiguous time interval for which the dominant scale $\tilde{s}(t)$ is within a statistically significant area in the timescale planethat is, being inside the cone of influence and having a significant $\mathrm{WCO}(\tilde{s}(t), t)>0.83$-as a coherent oscillation regime. The remaining time intervals constitute the non-coherent oscillation regimes. Each signal on its own may well oscillate in a regular fashion even within a non-coherent oscillation regime, but for a pair of signals the wavelet analysis guarantees a significantly correlated oscillation-and therefore a well-defined phase relationship-only in a coherent oscillation regime.

To characterize the typical duration $\widetilde{T}$ of coherent oscillations in an ensemble of signals, we first measure the length $T_{i}$ of each coherent oscillation regime $\mathrm{i}=1 \ldots N$ that occurs in the ensemble and then calculate the expected oscillation regime duration, $\widetilde{T}$, of a randomly chosen time instance taken from a coherent oscillation regime $\widetilde{T}=\sum_{i=1}^{N} T_{i}^{2} / \sum_{i=1}^{N} T_{i}$. We found that this measure gives a better description of the typical time to observe a coherent oscillation interval than the median or the mean $\bar{T}=\sum_{i=1}^{N} T_{i} /$ Nof the length distribution.

\section{Circular phase distribution}

To characterize the phase relationship between a pair of covarying signals, we calculated the dominant phase differences $\theta_{k}$ for all time instances within a coherent oscillation regime. We used circular statistics ${ }^{33}$ to characterize the resulting distribution of phase differences $\theta_{k}$. For this we calculated the complex order parameter $z$, which is

defined as the average of the complex numbers $\mathrm{e}^{i \theta_{k}}$

$$
z=R \mathrm{e}^{i \bar{\theta}}=\frac{1}{N} \sum_{k=1}^{N} \mathrm{e}^{i \theta_{k}}
$$

Here the phase angle $\bar{\theta}$ of $z$ indicates the circular mean of the phase differences, and the amplitude $R$ of $z$ gives the circular standard deviation of the distribution as $\sqrt{-2 \ln R}$.

Given the sampling frequency of $1 \mathrm{day}^{-1}$, the Nyquist frequency is $0.5 \mathrm{day}^{-1}$, which for an average period length of 6.7 days roughly corresponds to a phase resolution of $27^{\circ}$. Averaging phase differences over many cycles enabled us to achieve a much better phase resolution, yielding typically rather smooth distributions of phase differences. We verified that these smooth phase distributions are no artefacts, by comparing phase distributions obtained from our highly resolved numerical simulations (see 'Numerical simulation') with those obtained from time series sampled from these simulations once per day, which yielded very similar results.

\section{Phase signature}

To characterize the mutual phase relationship of not only two but also an ensemble of time series (for example, total densities of predator and prey and of measured life history stages), we calculated the distribution of phase differences of all time series with respect to one selected reference time series. The combined plot of all resulting circular distributions in one common polar histogram defines our 'phase signature', providing a fingerprint for the cyclic succession of the corresponding state variables.

\section{Parametrization and smoothing}

Following a previously published study ${ }^{31}$, we performed smoothing in time by convolution with a normalized real Morlet wavelet $\exp \left[-\tau^{2} /\left(2 s^{2}\right)\right] /(s \sqrt{2 \pi})$. Smoothing in scale direction was obtained by averaging over a constant-length (boxcar) window over 0.6 octaves-the scale decorrelation length for the Morlet wavelet-on a logarithmic scale $^{31}$. Similarly, we took the upper and lower scale band borders $s_{1}$ and $s_{2}$ as \pm 0.6 octaves from the peak in the global (that is, time-averaged) WCS. For a better visualization, circular histograms (bin width of $\pi / 100$ ) were smoothed by a moving average (window width $0.15 \pi$, the above calculated phase resolution).

We verified that the resulting phase distributions remain largely unchanged for different parameterizations of the wavelet analysis, in particular for different wavelet functions, different values of the wavelet parameter $\omega_{0}$, different smoothing parameters and scale band borders $s_{1}$ and $s_{2}$.

\section{Numerical simulation}

We developed a mathematical model to describe a stage-structured predator-prey community in a chemostat ${ }^{34,35}$, which closely follows our experimental setup. The model includes as state variables the concentration of nitrogen $N$, phytoplankton $P$, and three life stages of the predator, namely eggs $E$, juveniles $J$ and adults $A$. Additionally the model keeps track of the dead animals $D$ in the chemostat. We assume a constant time delay $\theta$ for egg development, a constant time delay $\tau$ for juvenile maturation, and the same constant mortality rate for juveniles and adults (deliberately keeping the model simple, as intake-related regulation $\mathrm{s}^{34,35}$ did not improve the agreement between simulation results and data). We assume that the phytoplankton population is unstructured. All variables are measured

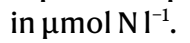

The model is written as the following system of time-delayed ordinary differential equations:

$$
\begin{aligned}
& \dot{N}=\delta N_{\text {in }}-F_{\mathrm{P}}(N) P-\delta N \\
& \dot{P}=F_{\mathrm{P}}(N) P-F_{\mathrm{B}}(P) B / \varepsilon-\delta P \\
& \dot{E}=R_{\mathrm{E}}-R_{\mathrm{J}}-\delta E \\
& \dot{J}=R_{\mathrm{J}}-R_{\mathrm{A}}-(m+\delta) J \\
& \dot{A}=\beta R_{\mathrm{A}}-(m+\delta) A \\
& \dot{D}=m(J+A)-\delta D
\end{aligned}
$$

Here, $\beta$ is the adult/juvenile mass ratio (the juvenile/egg mass ratio is assumed to be 1 ) and the recruitment rates are

$$
\begin{aligned}
& R_{\mathrm{E}}(t)=F_{\mathrm{B}}(P(t)) A(t) \\
& R_{\mathrm{J}}(t)=R_{\mathrm{E}}(t-\theta) \exp (-\delta \theta) \\
& R_{\mathrm{A}}(t)=R_{\mathrm{J}}(t-\tau) \exp (-\delta \tau)
\end{aligned}
$$

Algal nutrient uptake $F_{\mathrm{p}}(N)$ is modelled as a Monod function and predator recruitment as a type- 3 functional response with Hill coefficient $K$

$$
\begin{aligned}
& F_{\mathrm{P}}(N)=r_{\mathrm{P}} \frac{N}{K_{\mathrm{P}}+N} \\
& F_{\mathrm{P}}(P)=r_{\mathrm{B}} \frac{P^{K}}{K_{\mathrm{B}}^{K}+P^{K}}
\end{aligned}
$$

The total predator density is given by $B(t)=\beta J(t)+A(t)$ and the egg ratio is $\beta E(t) / B(t)$. As state variables are given in units of nitrogen, juveniles and eggs here need to be scaled by the adult/juvenile mass ratio $\beta$.

The model is solved numerically by an Euler scheme with a time step of $h=0.001$ days. We included environmental stochasticity by taking the growth rates $r_{\mathrm{p}}$ and $r_{\mathrm{B}}$ as autocorrelated random numbers by multiplying them in every time step $i$ with a factor of $1+s_{i}$. 
Autocorrelation (that is, red noise) is implemented as $s_{i+1}=a s_{i}+\xi_{i}$ (first-order autoregressive AR(1) process ${ }^{36}$ ) with autocorrelation parameter $a$ and independent and identically distributed Gaussian numbers $\xi_{i}$ with mean 0 and variance $\sigma^{2}\left(1-a^{2}\right)$. For wavelet analysis and for representation in figures, the simulated states are sampled with a rate of 1 per day, in accordance with the experiments. To capture measurement error and additional stochastic influences, we replaced the sampled rotifer state variables, $E(t), J(t), A(t)$ and $D(t)$, by random values drawn from a Poisson distribution with an expectation value equal to that of the state variable. Finally, algal and rotifer state variables were rescaled to individuals per volume using the constants $v_{\mathrm{P}}$, and $v_{\mathrm{B}}$, respectively.

Parameter values were taken as follows. Mass ratio adult/juveniles, $\beta=5$; dilution rate, $\delta=0.55$ per day; nitrogen concentration in the external medium, $N_{\text {in }}=80 \mu \mathrm{mol} \mathrm{I}^{-1}$; phytoplankton maximal growth rate, $r_{\mathrm{P}}=3.3$ per day; phytoplankton half-saturation constant: $k_{\mathrm{P}}=4.3 \mathrm{\mu mol}^{-1}$; rotifer maximal egg-recruitment rate, $r_{\mathrm{B}}=2.25 \mathrm{per}$ day; rotifer half-saturation constant, $k_{\mathrm{B}}=15 \mu \mathrm{mol} \mathrm{I}^{-1}$; Hill coefficient of the functional response, $k=1.25$; predator assimilation efficiency, $\varepsilon=0.25$; rotifer mortality rate, $m=0.15$ per day; egg development time, $\theta=0.6$ days; juvenile maturation time, $\tau=1.8$ days; autocorrelation parameter, $a=0.9$; noise strength, $\sigma=0.5$; nitrogen content per adult female Brachionus, $v_{\mathrm{A}}=0.57 \times 10^{-3} \mu \mathrm{mol} \mathrm{N}$ per female; nitrogen content per algal cell, $\nu_{\mathrm{P}}=28 \times 10^{-9} \mu \mathrm{mol} \mathrm{N}$ per cell.

Environmental parameters $\left(\delta, N_{\text {in }}\right)$ were taken according to the experimental set-up. Parameters for uptake and growth $\left(r_{\mathrm{p}}, k_{\mathrm{p}}, r_{\mathrm{B}}, k_{\mathrm{B}}\right.$ and $\varepsilon$ ) were taken according to a previous study ${ }^{16}$. The rotifer mortality rate $(m)$ was increased compared with this previous study ${ }^{16}$, as we did not include the loss of the reproducing rotifer cohort into senescent animals. The mass ratio of adults to juveniles $(\beta)$ was taken from the literature ${ }^{37}$. Duration of development stages $(\theta, \tau)$ was adjusted to be within the previously reported range ${ }^{38}$. We used a type- 3 functional response ${ }^{39,40}$. Only the parameter values of population dynamics $(\theta, \tau, \kappa$ and $m)$ and stochasticity (noise levels and noise colour) were adjusted by hand within ecologically reasonable ranges to simultaneously optimally fit the comprehensive experimental data. As criteria we used the observed range and variability of the state variables, existence of oscillatory dynamics with correct period length, phase signatures (that is, phase relationships between all state variables, including the egg ratio), power and wavelet spectra, and the typical duration of coherent oscillatory regimes-both in the unforced and forced scenarios.

\section{Reporting summary}

Further information on research design is available in the Nature Research Reporting Summary linked to this article.

\section{Data availability}

Experimental data are publicly available on Figshare (https://doi. org/10.6084/m9.figshare.10045976.v1).

\section{Code availability}

Data analysis and the model were implemented in the language Julia v.1.1 ${ }^{41}$. The source code for the wavelet analysis is publicly available on GitHub (https://github.com/berndblasius/WaveletAnalysis).

29. Torrence, C. \& Webster, P. J. Interdecadal changes in the ENSO-monsoon system. J. Clim. 12, 2679-2690 (1999).

30. Maraun, D. \& Kurths, J. Cross wavelet analysis: significance testing and pitfalls. Nonlin. Processes Geophys. 11, 505-514 (2004).

31. Si, B. C. Spatial scaling analyses of soil physical properties: a review of spectral and wavelet methods. Vadose Zone J. 7, 547-562 (2008).

32. Bandrivskyy, A., Bernjak, A., McClintock, P. \& Stefanovska, A. Wavelet phase coherence analysis: application to skin temperature and blood flow. Cardiovasc. Eng. 4, 89-93 (2004).

33. Batschelet, E. Circular Statistics in Biology (Academic, 1981).

34. McCauley, E., Nisbet, R. M., de Roos, A. M., Murdoch, W. W. \& Gurney, W. S. C. Structured population models of herbivorous zooplankton. Ecol. Monogr. 66, 479-501 (1996).

35. de Roos, A. M. \& Persson, L. Population and Community Ecology of Ontogenetic Development 59 (Princeton Univ. Press, 2013).

36. Massie, T. M., Weithoff, G., Kuckländer, N., Gaedke, U. \& Blasius, B. Enhanced Moran effect by spatial variation in environmental autocorrelation. Nat. Commun. 6, 5993 (2015).

37. Mourelatos, S., Pourriot, R. \& Rougier, C. Taux de filtration du rotifère Brachionus calyciflorus: comparaison des méthodes de mesure; influence de l'âge. Vie Milieu 40, 39-43 (1990).

38. Halbach, U. Einfluß der Temperatur auf die Populationsdynamik des planktischen Rädertieres Brachionus calyciflorus Pallas. Oecologia 4, 176-207 (1970).

39. Fussmann, G. E., Weithoff, G. \& Yoshida, T. A direct, experimental test of resource vs. consumer dependence. Ecology 86, 2924-2930 (2005).

40. Seifert, L. I. et al. Heated relations: temperature-mediated shifts in consumption across trophic levels. PLoS ONE 9, e95046 (2014).

41. Bezanson, J., Edelman, A., Karpinski, S. \& Shah, V. B. Julia: a fresh approach to numerical computing. SIAM Rev. 59, 65-98 (2017).

Acknowledgements This work was supported by German VW-Stiftung. We thank A. Bandrivskyy, T. Massie, E. Denzin and all other technical staff of the Department of Ecology \& Ecosystem Modelling; and R. Ceulemans, C. Feenders, A. Jamieson-Lane, A. Ryabov and E. van Velzen for comments on the manuscript.

Author contributions B.B., G.F.F., G.W. and U.G. designed the research, L.R., G.F.F. and G.W. performed the experiments, B.B. and L.R. performed the numerical simulations and data analysis, B.B. and G.F.F. wrote the paper. All authors discussed the results and commented on the manuscript.

Competing interests The authors declare no competing interests.

\section{Additional information}

Supplementary information is available for this paper at https://doi.org/10.1038/s41586-0191857-0.

Correspondence and requests for materials should be addressed to B.B.

Peer review information Nature thanks Alan Hastings and the other, anonymous, reviewer(s)

for their contribution to the peer review of this work.

Reprints and permissions information is available at http://www.nature.com/reprints. 

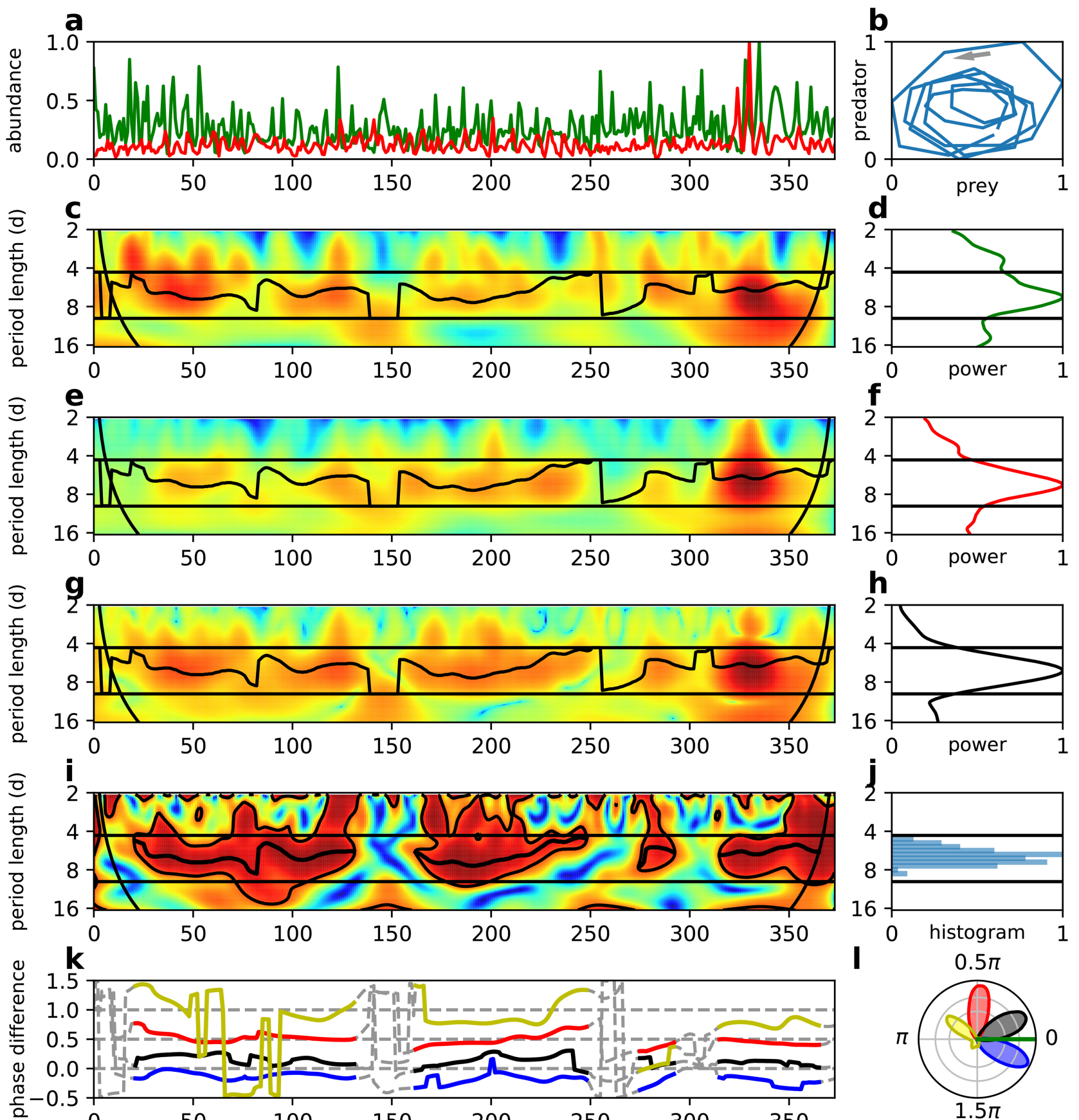

\section{Extended Data Fig. 1 | Detailed dynamics and phase relationship in}

experiment C1.a, Time series of the predator, B. calyciflorus (red), and its prey, M. minutum (green), (normalized to the range of 0 and 1$)$. b, Phase portrait of the bandpass-filtered predator-prey time series between days 90 and 130 . The arrow indicates the direction of oscillation. c, Local WPS for the prey, ranging from 0 (blue) to maximum (red), and the instantaneous oscillation period $\tilde{s}(t)$ (black line) of the highest WCO within the prefixed period length band (horizontal black lines). The cone of influence is also indicated (black). d, Global WPS $P(s)$ for the prey (green). Horizontal lines as in c.e, $\mathbf{f}$, As in c, d, but for the predator. $\mathbf{g}$, WCS between predator and prey, colour-coding and other lines as in c. h, Amplitude of the global WCS for the predator and the prey. i, WCO between predator and prey, ranging from 0 (blue) to 1 (red). Significant areas $(\mathrm{WCO}>0.83)$ are enclosed by thin solid lines; the thick black line segments show the instantaneous oscillation period $\tilde{s}(t)$ in these segments. $\mathbf{j}$, Histogram of period lengths within coherent oscillation regions as detected in i. k, Phase difference $\theta(t)$ to prey for the predator (red), the predator egg ratio (blue), the number of eggs (black) and dead animals (yellow). Significant relations between respective signals are marked as a solid line. I, Circular distribution of the mutual phase difference to prey (indicated in green) for the predator (red), the predator egg ratio (blue), the number of eggs (black) and dead animals (yellow). 

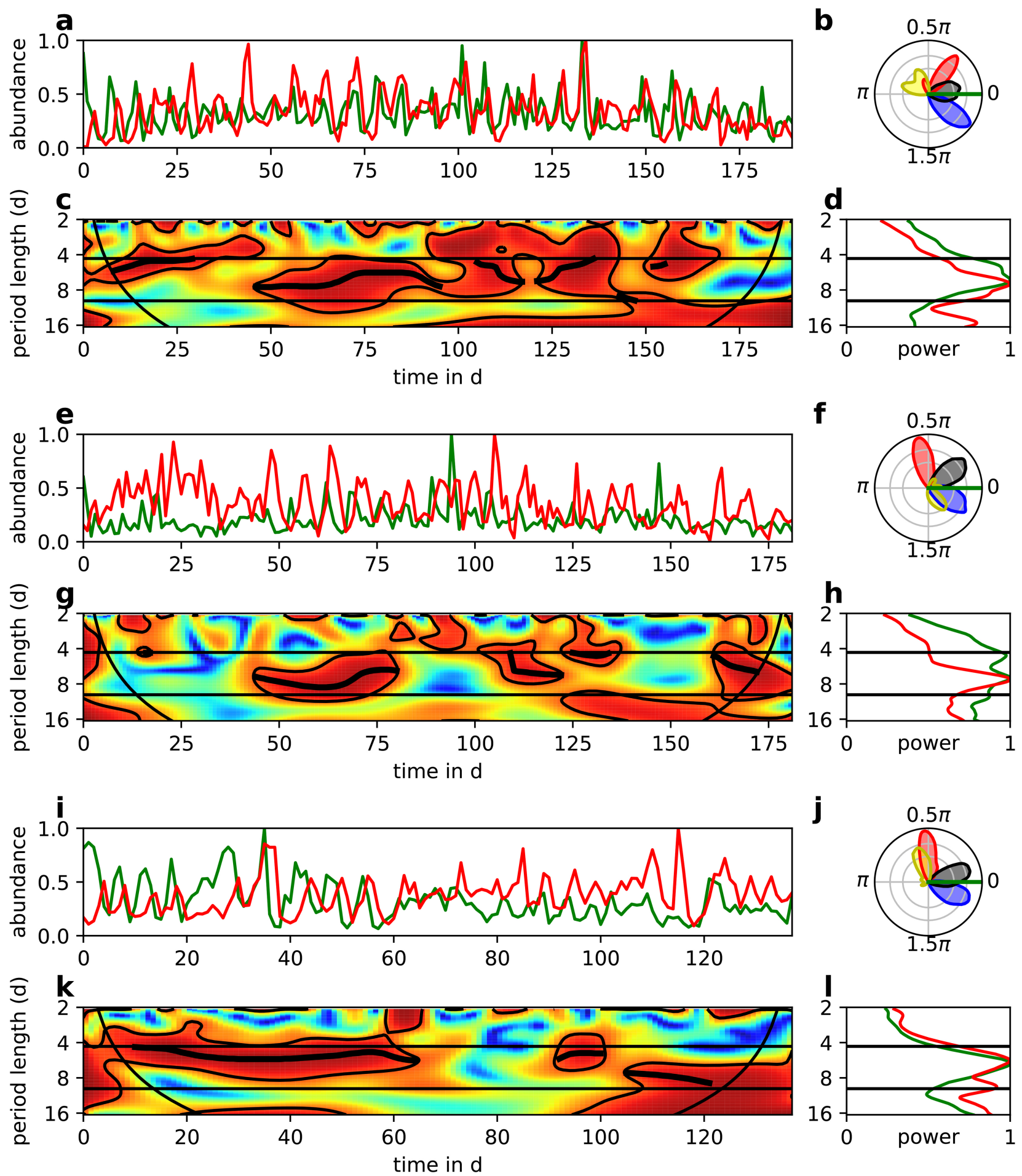

Extended Data Fig. 2 | Dynamics and phase relationships in three further experimental time series in a constant environment. a-d, Analysis of experiment C2. a, Predator-prey time series.b, Phase signature.c, WCO.d,
Global WPS. e-h, As in a-d, for experiment C3. i-l, As in a-d, for experiment C4. Details and colours as in Fig. 1 . 

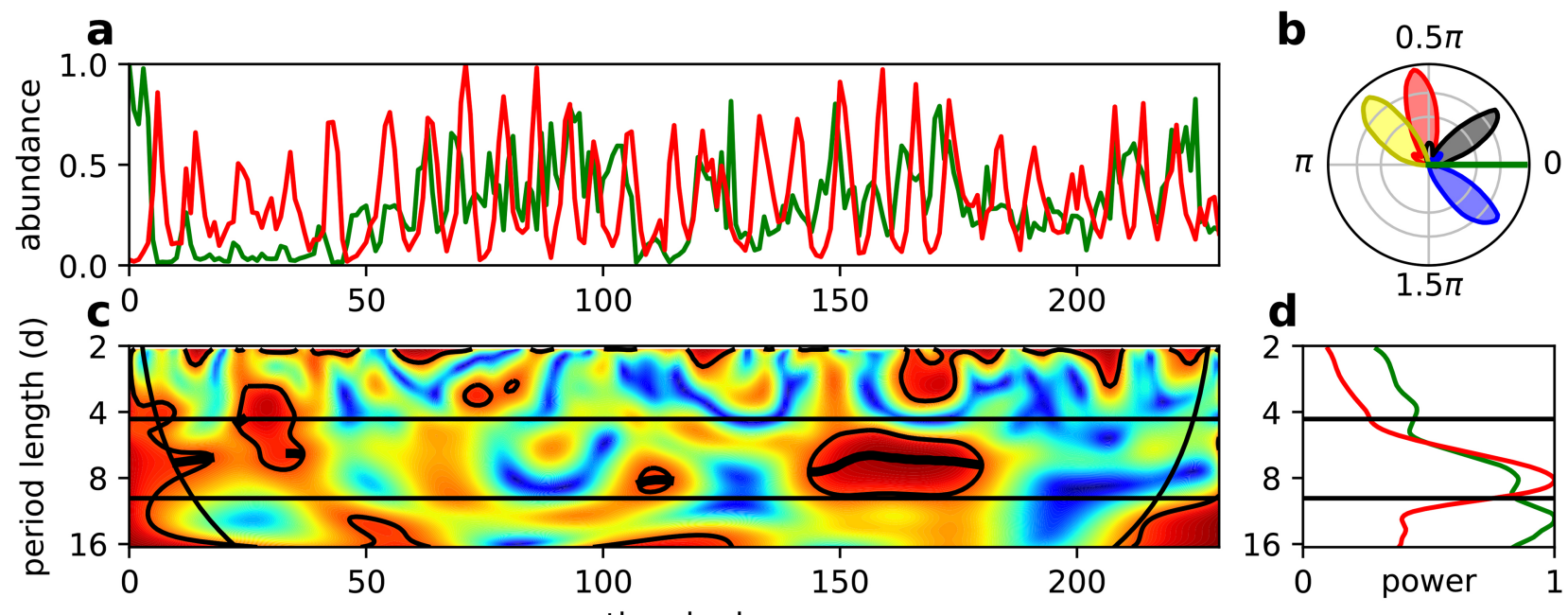

d
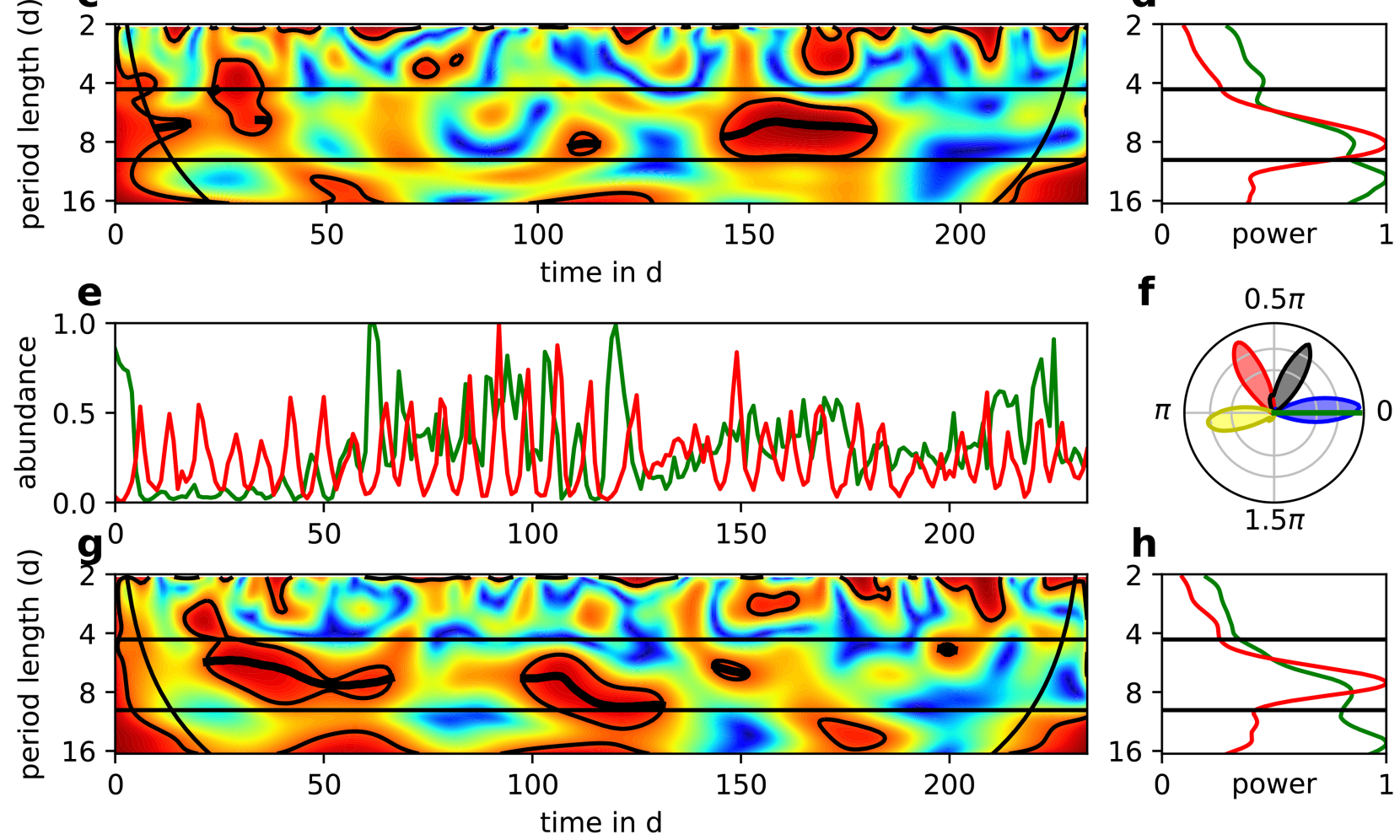

Extended Data Fig. 3 | Dynamics and phase relationships in measured time series with a different algal species (C. vulgaris). a-d, Analysis of experiment C6. a, Predator-prey time series.b, Phase signature. c, WCO.d, Global WPS. e-h, As in a-d, for experiment C7. Details and colours as in Fig. 1. 

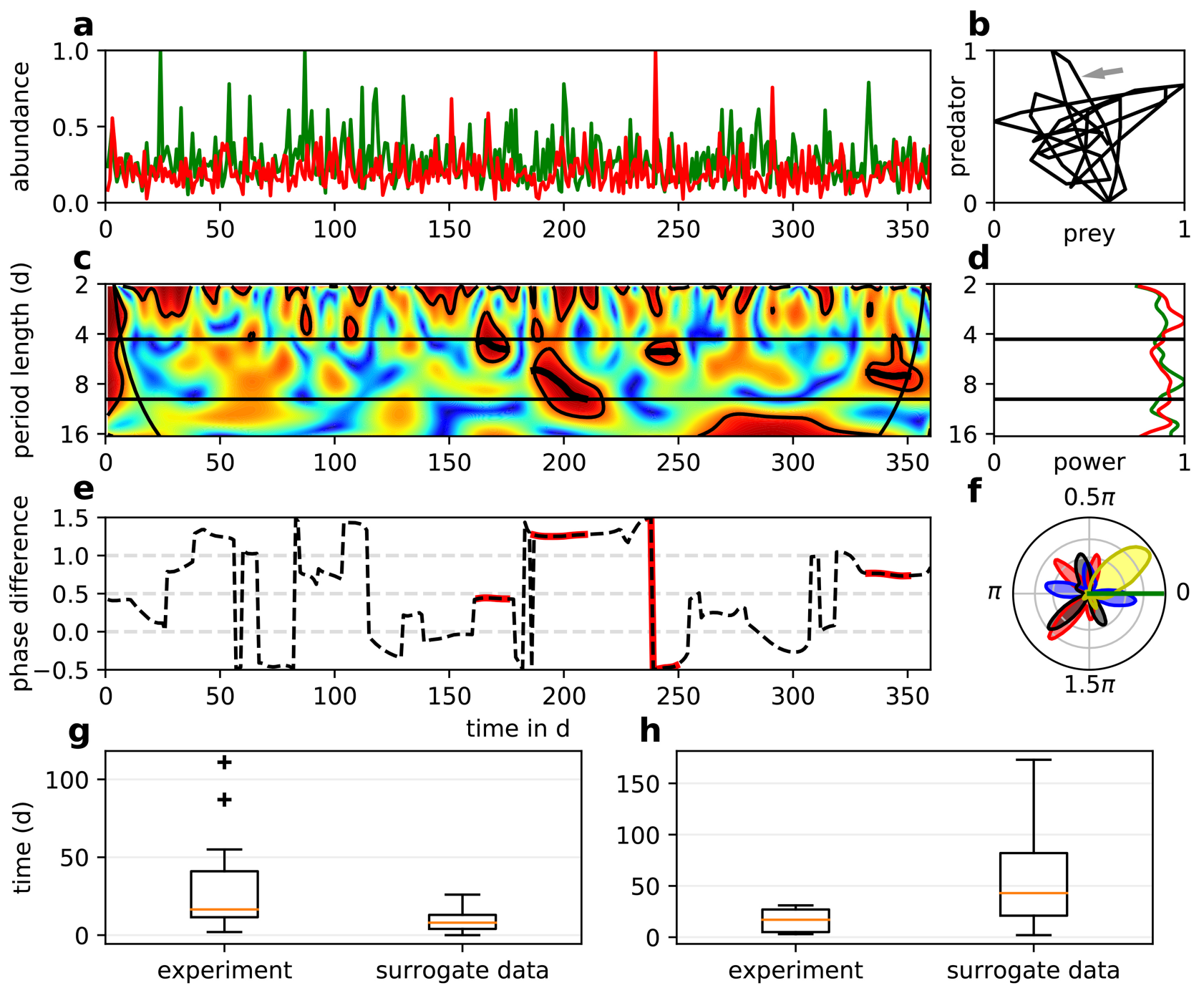

Extended Data Fig. 4 | Dynamics and phase relationship in surrogate data and length distribution of coherent oscillation regimes. a-f, As in Fig. 1, but for surrogate data, obtained by time scrambling-that is, randomly drawing 360 values with replacement from the predator and prey time series in experiment $\mathrm{C} 1$-yielding a time series without temporal correlation (white noise) but with the same abundance distribution as the experimental data. The figure shows a typically outcome. Occasionally, for short bursts the randomized predator and prey time series oscillate with a common frequency (a), yielding areas of high coherence (c; $\mathrm{WCO}>0.83$; spurious coherent oscillation regimes) with random phase differences (e). Whereas most time intervals with coherent predator-prey cycles in the surrogate data have a short duration, in this example (by chance) there is one regime (days 187-209) with a duration of 23 days. $\mathbf{g}, \mathbf{h}$, Box plots of oscillation regime length. Boxes show the interquartile range, orange lines indicate the median, whiskers range from the lowest to the highest data point within $1.5 \times$ the interquartile range and markers indicate outliers. $g$, Length distribution of coherent oscillation regimes for the

experimental data under free-running conditions (C1-C5) and for a large ensemble of surrogate data. This shows that time intervals with coherent oscillations are significantly longer in the experiments ( $n=20$; median, 16 days; mean \pm s.d., $29 \pm 28$ days) than in the surrogate data ( $n=13,719$; median, 8 days; mean \pm s.d., $9 \pm 7$ days). The two outliers in the left box plot correspond to the two long coherent oscillation regimes of 111 days and 85 days in $\mathrm{C} 1$, which (even though they are outliers in the length distribution) effectively dominate the dynamic behaviour in $\mathrm{C}$. Consequently, we calculate the typical duration $\widetilde{T}$ of coherent oscillations as the expected regime duration for a randomly chosen time instance taken from a coherent oscillation regime (Methods) $(\widetilde{T}=58$ days in $\mathrm{C} 1-\mathrm{C} 5 ; \widetilde{T}=15$ days in the surrogate data). h, As in $\mathbf{g}$, but for durations of noncoherent oscillation regimes, showing that time intervals of non-coherent oscillations are significantly shorter in the experiments ( $n=15$; median, 17 days; mean \pm s.d., $16 \pm 11$ days; typical duration $\widetilde{T}=23$ days) than in the surrogate data ( $n=12,719$; median, 43 days, mean \pm s.d., $60 \pm 11$ days; typical duration $\widetilde{T}=113$ days). 

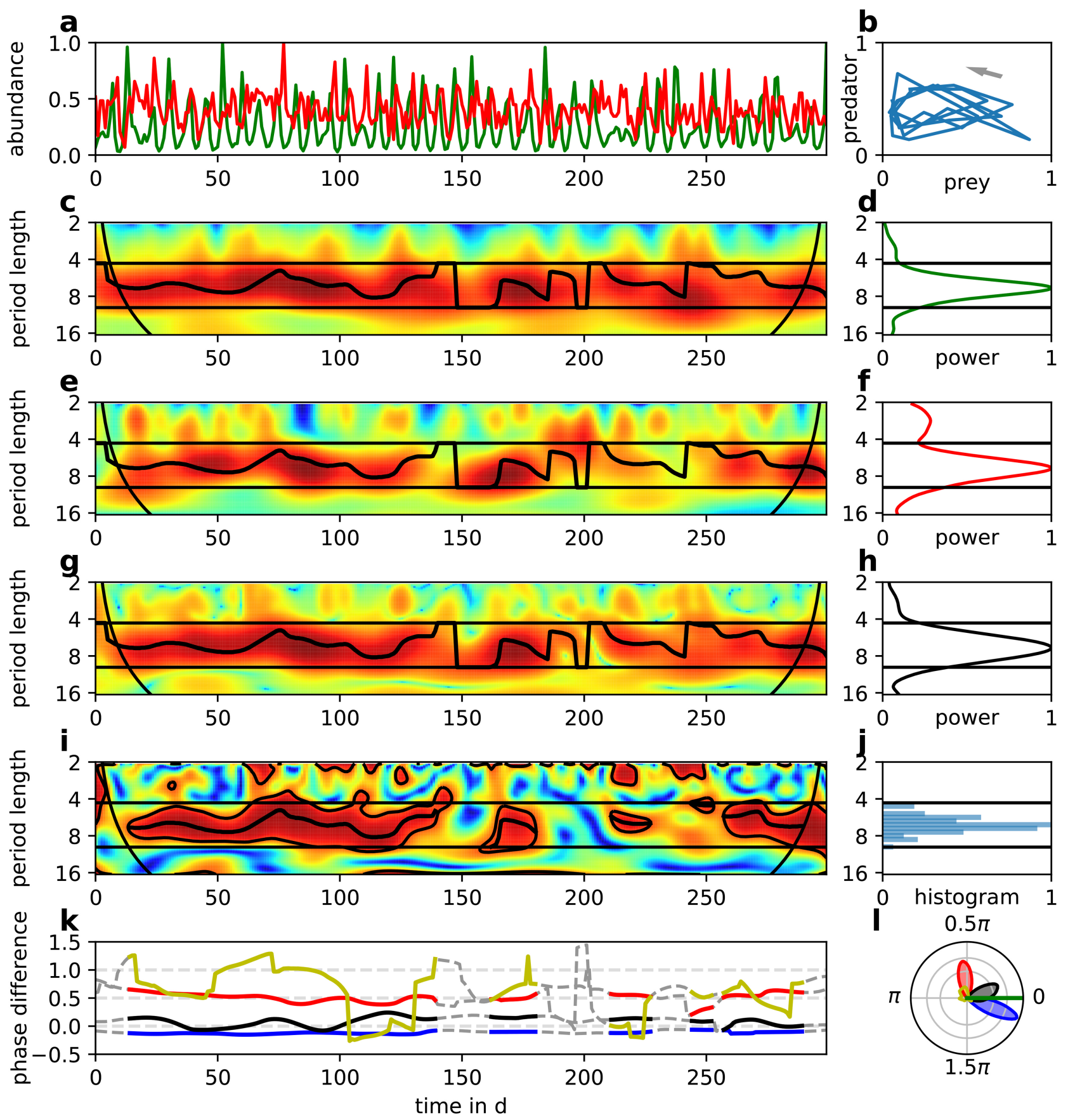

Extended Data Fig. 5 | Detailed dynamics and phase relationships in a modelled time series. a-I, As in Extended Data Fig. 1, but for a simulated time series of the stochastic time-delayed model in a constant environment. The simulation time was 300 days. A typical model outcome is shown. In this example, we obtained five coherent oscillation regimes (indicated as thick lines in $\mathbf{i}, \mathbf{k})$. Similar to the experimental systems $(\mathrm{C} 1-\mathrm{C} 7)$, these regimes are interspersed by short non-coherent oscillation regimes without a significant phase relationship. 


\section{a}

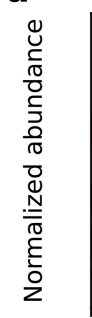

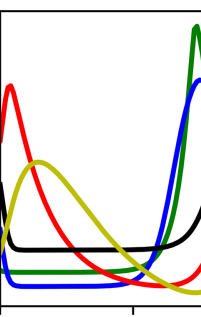

0

C

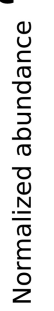

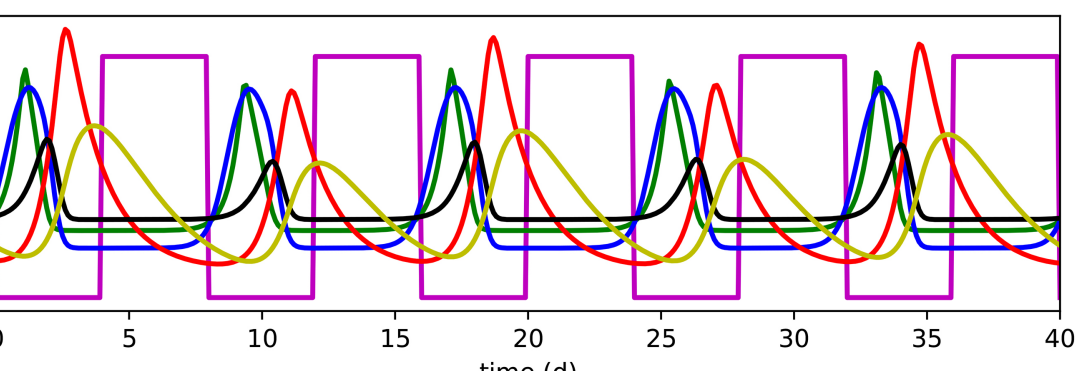

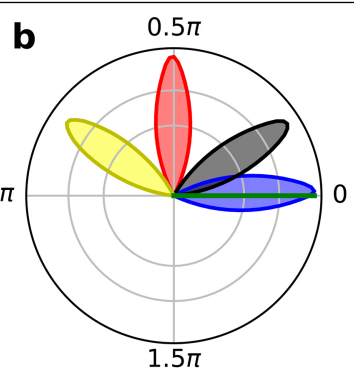

d

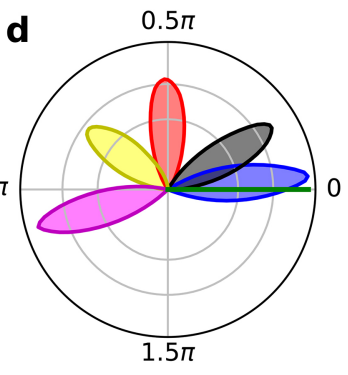

Extended Data Fig. 6 | Dynamics and phase relationships in a model without juvenile maturation delay. a, Simulated time series of algae (green), rotifers (red), eggs (black), egg ratio (blue) and dead animals (yellow) in a model without juvenile maturation delay, $\tau=0$, under constant conditions with all stochasticity removed (mean centred, relative units). b, Phase signature obtained from stochastic simulations (free-running conditions, $\tau=0$ ). c, d, As in $\mathbf{a}, \mathbf{b}$, but for an externally driven system (external nutrient concentration shown in magenta). The figure shows that in a scenario without juvenile maturation delay $(\tau=0)$, the phase of the egg ratio coincides with that of the algae, both in a free-running and in an externally driven model. This is in contrast to the experimental observations (Fig. 3, rows 1 and 3) and simulations with non-vanishing juvenile maturation delay ( $t=1.8$ days) (Fig. 3 rows 2 and 4 , Extended Data Fig. 9d, f), in which the egg ratio is significantly preceding the algae signal. Thus, the phase signature helps to disentangle life-history mechanisms, as-by comparison of observed and simulated phase signatures-a delay of juvenile maturation is essential to attain the experimentally observed phase relations. 


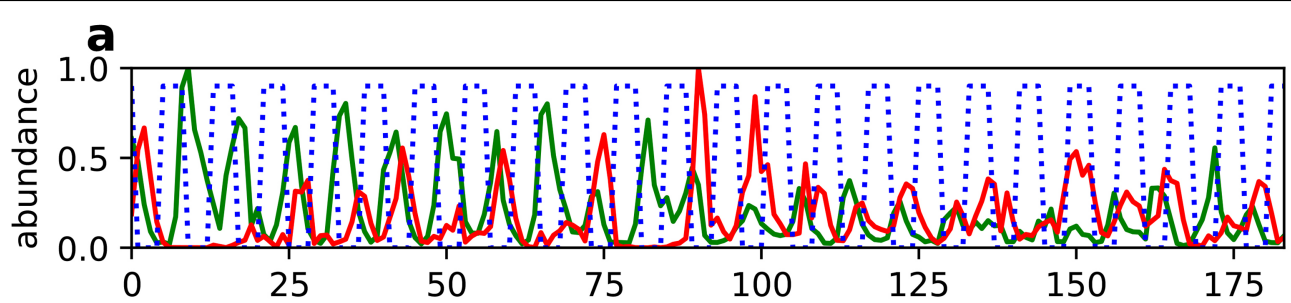

b
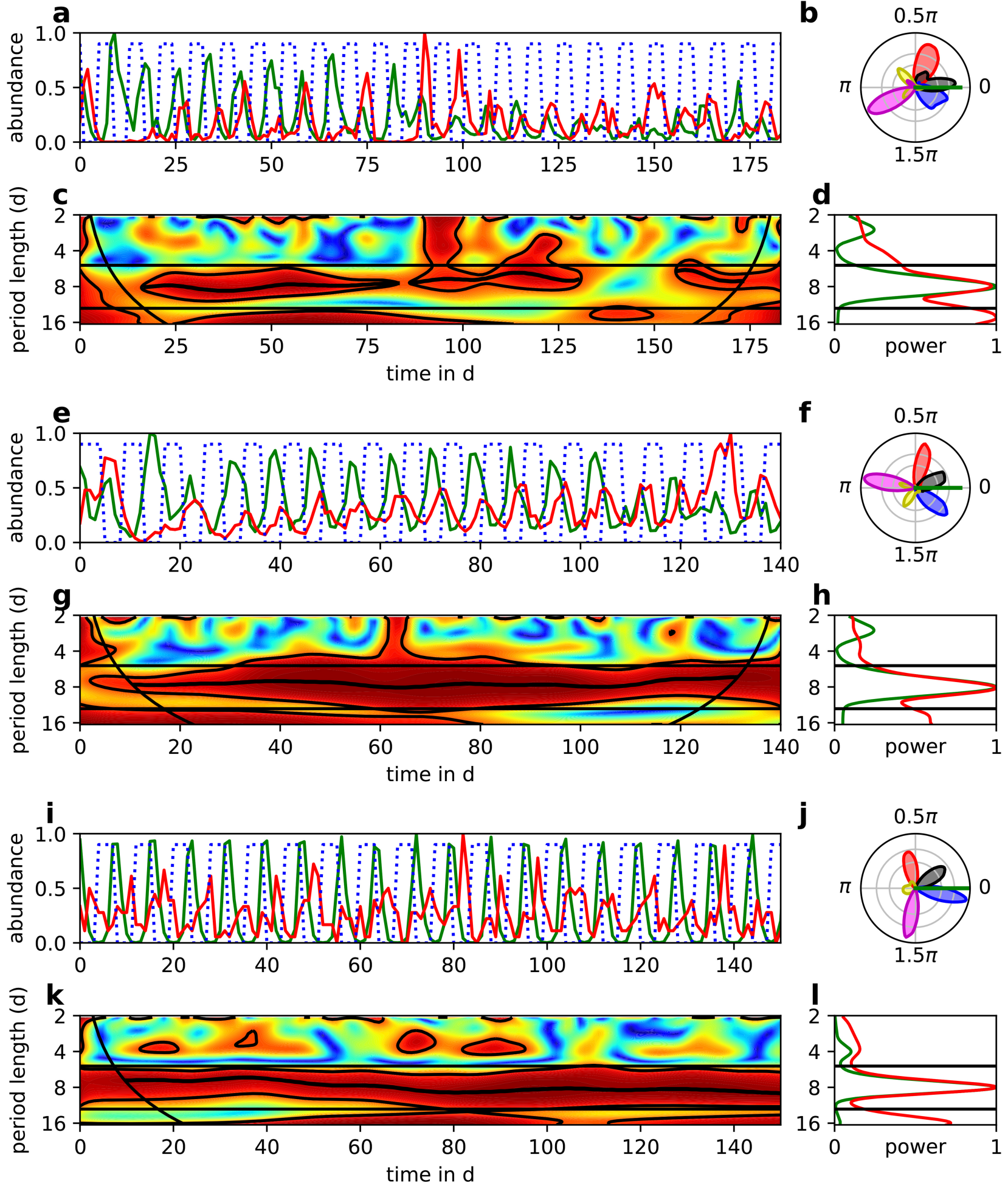

Extended Data Fig. 7 |Dynamics and phase relationships in externally driven systems. a-d, Analysis of experiment C8. a, Predator-prey time series. b, Phase signature.c, WCO.d, Global WPS. e-h, As in a-d, for experiment C9. i-l, As in

as in Fig. 1. In a, e, $\mathbf{i}$, the blue dotted line shows the input concentration in the external medium in normalized units. In $\mathbf{b}, \mathbf{f}, \mathbf{j}$, the phase difference distribution between the external medium and the prey is shown in magenta.

a-d, for simulation results of an externally driven system. Details and colours 

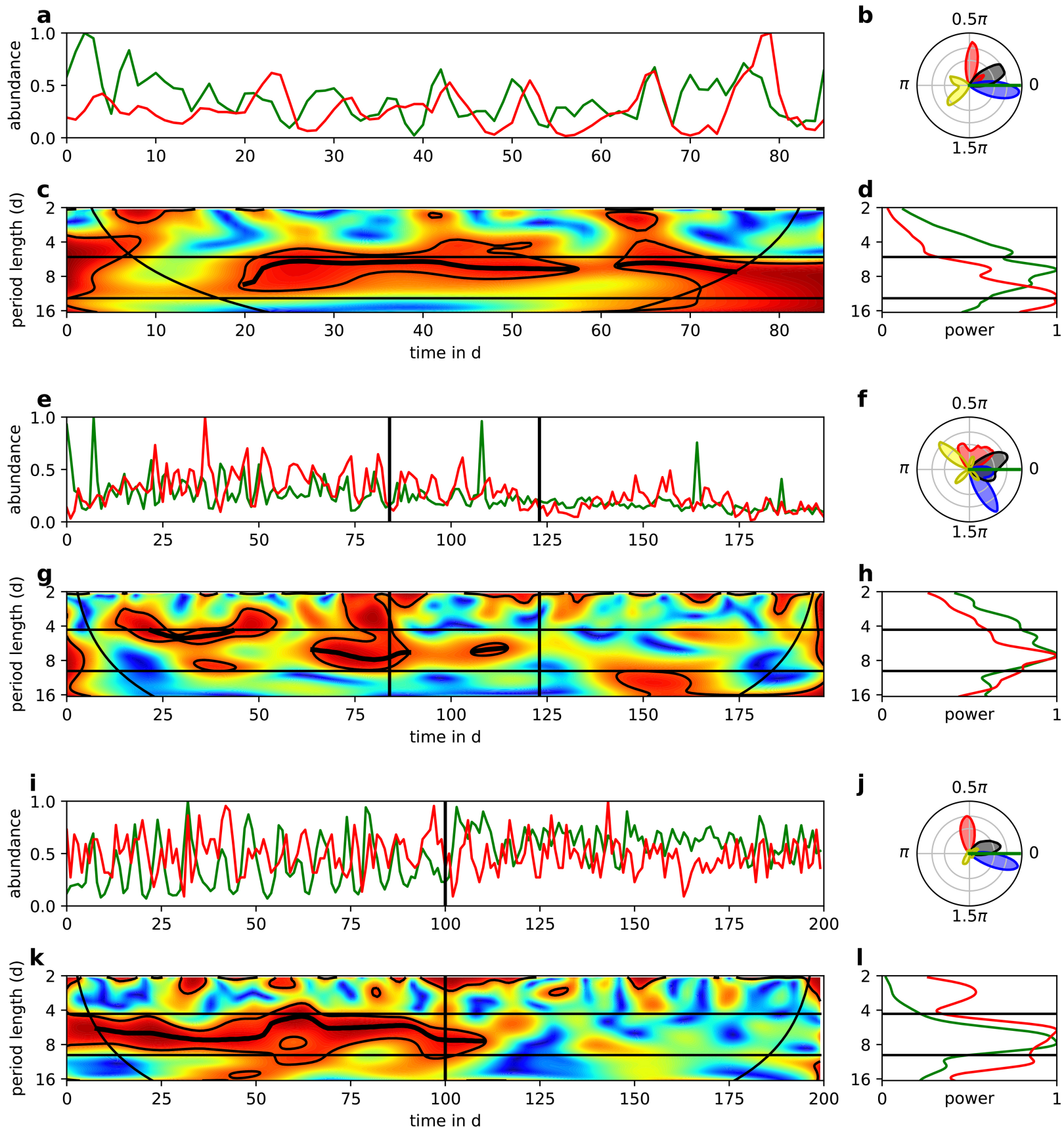

Extended Data Fig. 8 Dynamics and phase relationships in experiments and simulations with changed dilution rate and press perturbations. $a-d$, Analysis of experiment $\mathrm{C} 5$, which has an increased dilution rate $(\delta=0.66 \mathrm{per}$ day). a, Predator-prey time series. b, Phase signature.c, WCO.d, Global WPS. e-h, As in a-d, for experiment $\mathrm{C} 10$, which undergoes press perturbations (vertical black lines) at day 84 (dilution rate was increased from $\delta=0.55$ per day to $\delta=1.2$ per day) and day 123 (dilution rate increased further to $\delta=1.35$ per day). $\mathbf{i}-\mathbf{l}$, As in $\mathbf{a}-\mathbf{d}$, with simulation results of a system undergoing a press perturbation in which the dilution rate was increased to $\delta=0.75$ per day at day 100 (vertical black line in $\mathbf{i}, \mathbf{k}$ ). Under conditions of the increased dilution rate, the oscillations are suppressed (Extended Data Fig. 9a). Details and colours as in Fig. 1. 


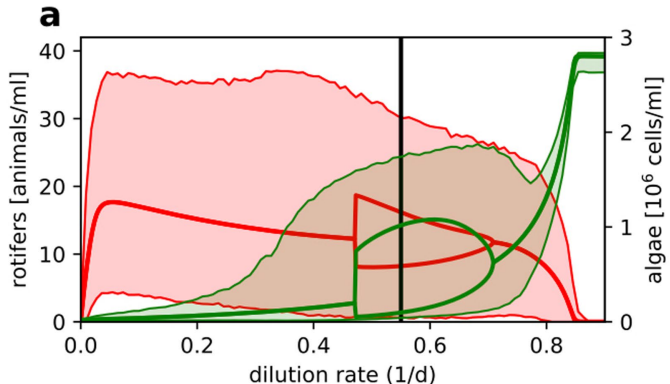

C
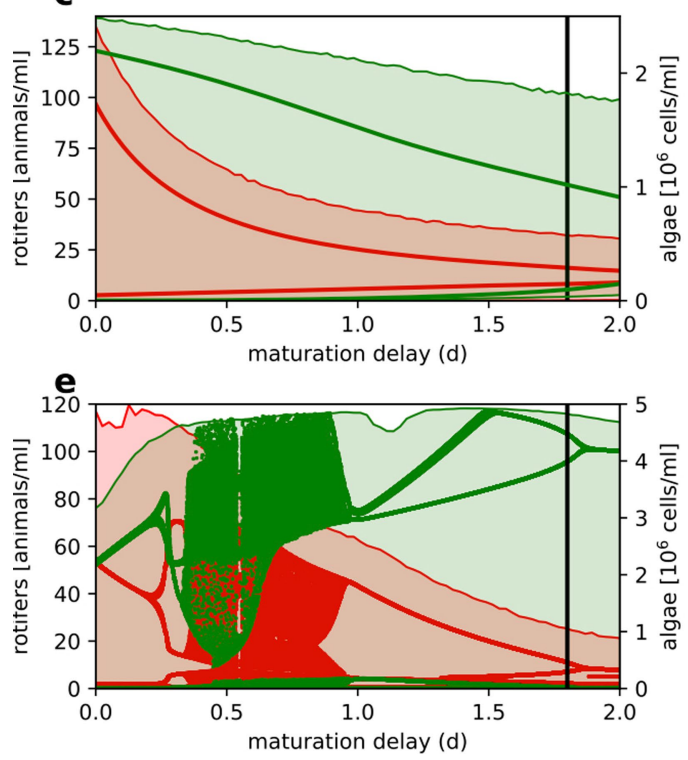

Extended Data Fig. 9|Bifurcation diagrams. a, Simulated values of predator (red) and prey (green) for different values of the dilution rate. Thick solid lines show maximal and minimal values obtained in the unforced model without stochasticity. Shaded areas show range of values in the stochastic model. Sustained predator-prey oscillations appear for $0.47<\delta<0.71$ per day. For large values of the dilution rate, $\delta>0.87$ per day, the predator goes extinct. $b$, As in a, but for the externally forced system. The model shows oscillations for all parameters without predator extinction $\delta<0.8$ per day, it exhibits a bistability regime (small- versus large-amplitude oscillations) for $0.23<\delta<0.31$ per day and a period-2 oscillation regime for $0.42<\delta<0.57$ per day. $\mathbf{c}, \mathbf{d}$, Bifurcation diagrams of the unforced model as a function of the

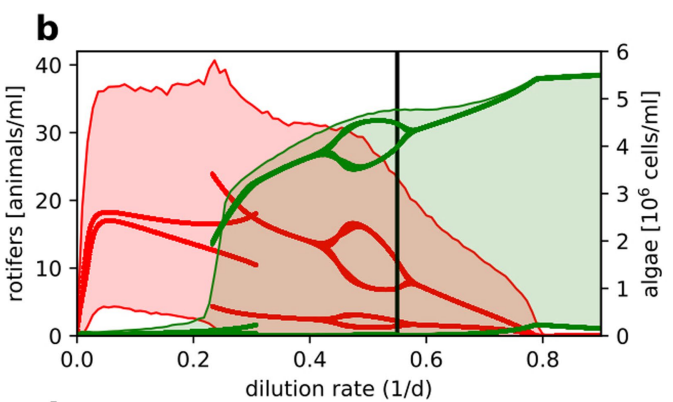

d
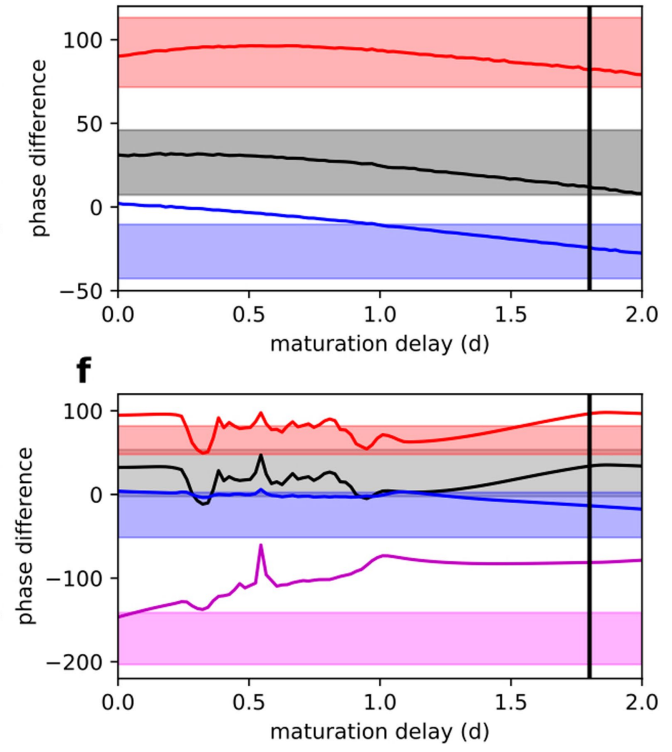

juvenile maturation delay $\tau$.c. As in a, but for the different bifurcation parameter.d, Phase difference to the algae for the predator (red), eggs (black) and the egg ratio (blue). The shaded areas indicate the range of phase differences $( \pm 1$ s.d.) from the experiments in a constant environment (C1-C5). For large values of $\tau$, the simulated phase signature aligns with the experimental data, whereas for small values of $\tau$, the simulated phase difference of the egg ratio approaches zero. e, f, As in $\mathbf{c}, \mathbf{d}$, but for the externally forced model.e, For small delay times, $0.4<\tau<1$ day, the model exhibits a chaotic regime.f, Additionally the phase difference between algae and external nutrients is shown (magenta). Vertical solid lines show the actually used parameter values $\delta=0.55$ per day and $\tau=1.8$ days. 
Extended Data Table 1 | Summary of all experiments

\begin{tabular}{|l|l|l|l|l|l|}
\hline Code & Experimental conditions & Algal species & Duration (days) & $N_{i}\left(\mu \mathrm{mol} \mathrm{I}^{-1}\right)$ & $\delta\left(\mathrm{day}^{-1}\right)$ \\
\hline C 1 & constant environment & M. minutum & 373 & 80 & 0.55 \\
\hline C 2 & constant environment & M. minutum & 189 & 80 & 0.55 \\
\hline C 3 & constant environment & M. minutum & 181 & 80 & 0.55 \\
\hline C 4 & constant environment & M. minutum & 137 & 80 & 0.55 \\
\hline C 5 & constant environment & M. minutum & 85 & 80 & 0.66 \\
\hline C 6 & constant environment & C. vulgaris & 230 & 160 & 0.55 \\
\hline C 7 & constant environment & C. vulgaris & 233 & 160 & 0.55 \\
\hline C 8 & externally forced & M. minutum & 183 & $0 / 160$ & 0.55 \\
\hline C 9 & externally forced & M. minutum & 140 & $0 / 160$ & 0.55 \\
\hline C 10 & press perturbation & M. minutum & 197 & 80 & $0.55 / 1.2 / 1.33$ \\
\hline
\end{tabular}

A series of ten chemostat experiments was performed, constituting a total of 1,948 measurement days (corresponding to 5.3 years of measurement) and covering 3 scenarios. (1) Constant

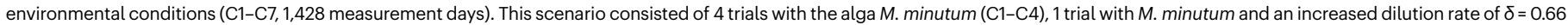

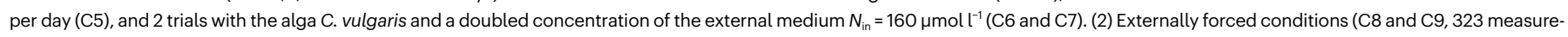
ment days). The concentration of the external medium was periodically changed between $N_{\text {in }}=160 \mu$ mol l $l^{-1}$ and $N_{\text {in }}=0 \mu$ mol l $l^{-1}$ with a period length of 8 days. (3) Press perturbation (C10, 197

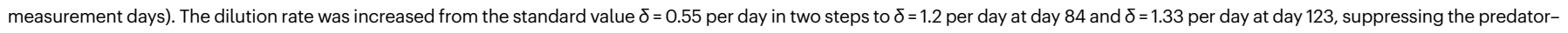
prey oscillations. 


\section{nature research}

Corresponding author(s): Bernd Blasius

Last updated by author(s): Oct 25, 2019

\section{Reporting Summary}

Nature Research wishes to improve the reproducibility of the work that we publish. This form provides structure for consistency and transparency in reporting. For further information on Nature Research policies, see Authors \& Referees and the Editorial Policy Checklist.

\section{Statistics}

For all statistical analyses, confirm that the following items are present in the figure legend, table legend, main text, or Methods section.

n/a Confirmed

\The exact sample size $(n)$ for each experimental group/condition, given as a discrete number and unit of measurement

$\bigotimes$ A statement on whether measurements were taken from distinct samples or whether the same sample was measured repeatedly

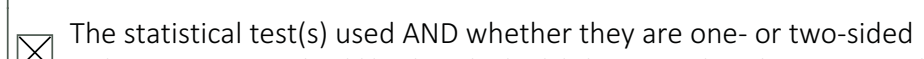

Only common tests should be described solely by name; describe more complex techniques in the Methods section.

$\searrow$ A description of all covariates tested

$\bigotimes$ A description of any assumptions or corrections, such as tests of normality and adjustment for multiple comparisons

A full description of the statistical parameters including central tendency (e.g. means) or other basic estimates (e.g. regression coefficient)

$\triangle$ AND variation (e.g. standard deviation) or associated estimates of uncertainty (e.g. confidence intervals)

For null hypothesis testing, the test statistic (e.g. $F, t, r$ ) with confidence intervals, effect sizes, degrees of freedom and $P$ value noted

$\triangle$ Give $P$ values as exact values whenever suitable.

Х $\square$ For Bayesian analysis, information on the choice of priors and Markov chain Monte Carlo settings

Х $\square$ For hierarchical and complex designs, identification of the appropriate level for tests and full reporting of outcomes

Х $\square$ Estimates of effect sizes (e.g. Cohen's $d$, Pearson's $r$ ), indicating how they were calculated

Our web collection on statistics for biologists contains articles on many of the points above.

\section{Software and code}

\section{Policy information about availability of computer code}

Data collection Measured data were first transfered to Excel-files (Versions 9-14) and subsequently archieved as csv-files.

Data analysis Data analysis and the model were implemented in the language Julia 1.1

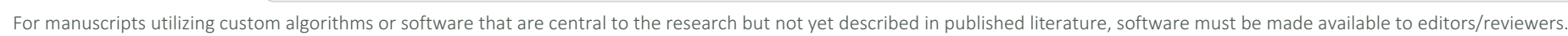
We strongly encourage code deposition in a community repository (e.g. GitHub). See the Nature Research guidelines for submitting code \& software for further information.

\section{Data}

Policy information about availability of data

All manuscripts must include a data availability statement. This statement should provide the following information, where applicable:

- Accession codes, unique identifiers, or web links for publicly available datasets

- A list of figures that have associated raw data

- A description of any restrictions on data availability

Experimental data were made publicly available on Figshare - doi.org/10.6084/m9.figshare.10045976.v1. Data analysis and the model were implemented in the language Julia 1.1 (ref. 41). The source code for the wavelet analysis was made publicly available on https.//github.com/berndblasius/WaveletAnalysis.

\section{Field-specific reporting}

Please select the one below that is the best fit for your research. If you are not sure, read the appropriate sections before making your selection. 


\section{Ecological, evolutionary \& environmental sciences study design}

All studies must disclose on these points even when the disclosure is negative.

Study description

The aim of the study was to generate and analyse experimental long term data. Thus, the experimental design differs from the typical (multi-)factorial design. From our time series, the relevant parameters were extracted. We had four replicates for the core long term experiments. Treatment factors were nutrient supply (constant vs. pulsed), type of algae (Monoraphidium vs Chlorella) and flow rate (shift from standard flow rate to high rates). These were run in duplicate or triplicate, which is common for such long-term experimental studies (see Extended Data Table 1). Each run represents an individual independent chemostat vessel. Each vessel contained $800 \mathrm{ml}$ culture medium. All experiments were run under stable conditions at $23^{\circ} \mathrm{C}$ and permanent illumination.

Research sample

The experimental algae were Monoraphidium minutum (SAG-243-1, Göttingen, Germany) and Chlorella vulgaris (UTEX 26, Texas, USA). Stock cultures were kept under non-limiting conditions to avoid synchronization of the cells. Animals were Brachionus calyciflorus, strain IGB, grown in stock cultures with the experimental algae under the same conditions. All species are cosmopolitan species occuring in various types of freshwaters. During the experiments, a daily sample of $8 \mathrm{ml}$ was taken, representing $1 \%$ of the total experimental volume. This sample size is large enough to quantify the number of algae and animals (see below) and small enough in order not to change the flow rate.

Sampling strategy

The sample volume was constant throughout the study and chosen according to the expected number of algae/rotifers within the sample. Each experimental vessel contained up to several thousands of rotifers and up to several billions of algae. Since turbulent mixing prevents spatial heterogeneity both species are randomly distributed. A sample of $8 \mathrm{ml}$ was taken $(5 \mathrm{ml}$ for the analysis of animals, $3 \mathrm{ml}$ for the analysis of algae). The maximum abundance of the animals is higher than $100 / \mathrm{ml}$ so that a $5 \mathrm{ml}$ sample is representative. As for the algae, the abundance is much higher (mio/ml), a smaller sub-sample was analysed using an electronic particle counter (CASY, Schärfe, Germany). Three sub-samples of $400 \mu l$ were analysed and the mean was calculated. The coefficient of variation was on average 0.015 .

Data collection

Data were collected daily, mostly by E. Denzin, or other staff members, guided by G. Weithoff and G. Fussmann. Each person who participated in sampling was individually trained to avoid sampling bias. The same holds for the quantitative analysis of algae and rotifers. The raw data from the electronic particle counter were archived on a computer and also transferred to Excel-files (Versions 9 - 14). The data from the microscopic quantification of the animals were first recorded on paper in a laboratory folder and then transferred to Excel-files (Versions 9 - 14). The correct transfer was doublechecked in order to avoid any error through the transfer process. From these files, csv-files were created and used further onwards.

Timing and spatial scale The first experiments started in April 2001 and lasted until May 2009; data acquisition (counting) and analyses extended until 2019. Depending on human and spatial resources up to four chemostats were run simultaneously, often only two. The sampling frequency of daily samples was chosen in accordance to the growth rate of the experimental organisms. Daily samples allow for capturing the dynamic population behavior of the organisms and the subsequent analysis of population oscillations.

Data exclusions

Experimental runs were discarded from the data set when technical problems (leakage of tubes, blackout of pumps or illumination etc.) occurred before the data set was sufficiently long to extract the key parameters for this study.

Reproducibility

The reproducibility is part of the study, demonstrating that the findings were recurrent in several independent experimental runs.

Randomization

Treatments were set up independently and placed randomly in the climate chamber or different experimental runs, which were run subsequently, were run at the same spot.

Blinding

Blinding was neither possible, nor necessary. The correct type of medium, and flow rate had to be visible to the experimentalists.

Did the study involve field work? $\square$ Yes $\bigotimes$ No

\section{Reporting for specific materials, systems and methods}

We require information from authors about some types of materials, experimental systems and methods used in many studies. Here, indicate whether each material, system or method listed is relevant to your study. If you are not sure if a list item applies to your research, read the appropriate section before selecting a response.

Materials \& experimental systems

Methods

$\mathrm{n} / \mathrm{a}$ Involved in the study

$\mathrm{n} / \mathrm{a}$ Involved in the study

Х $\square$ Antibodies

Х $\square$ Eukaryotic cell lines

Х $\square$ ChIP-seq

Х Palaeontology

X $\square$ Flow cytometry

\ $\square$ Animals and other organisms

X $\square$ MRI-based neuroimaging

\ $\square$ Human research participants

\ $\square$ Clinical data 